\title{
Micro-to-macro transitions for heterogeneous material layers accounting for in-plane stretch
}

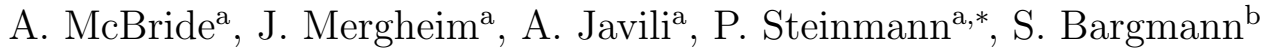 \\ a Chair of Applied Mechanics, University of Erlangen-Nuremberg, Egerlandstr. 5, \\ 91058 Erlangen, Germany, Tel.: +49 (0)913185 28502, Fax: +49 (0)91318528503 \\ ${ }^{b}$ Institute of Mechanics, TU Dortmund, Leonhard-Euler-Str. 5, 44227 Dortmund, \\ Germany, Tel.: +49(0)231755 7902
}

\begin{abstract}
The computational micro-to-macro transition framework couples heterogeneities on the microscopic scale to the macroscopic response of a continuum. The objective here is to apply this framework to macroscopic material layers capable of undergoing an in-plane stretch in addition to the normal opening mode. This is achieved using the continuum interface theory of Gurtin and Murdoch (1975) which endows the interface with its own energetic structure. The relation of the macroscopic kinematic descriptors of the interface deformation to the averaged underlying microscopic quantities are consistently derived using the Hill-type averaging theorem. Key features of the theory are elucidated using a series of three-dimensional numerical examples.
\end{abstract}

Keywords: micro-to-macro transitions, A. microstructures, B. constitutive behaviour, C. finite elements

\footnotetext{
*Corresponding author

Email addresses: andrew.mcbride@ltm.uni-erlangen.de (A. McBride), julia.mergheim@ltm. uni-erlangen.de (J. Mergheim), ali.javili@ltm. uni-erlangen.de (A. Javili), paul.steinmann@ltm. uni-erlangen.de (P. Steinmann), swantje.bargmann@tu-dortmund.de (S. Bargmann) Preprint submitted to Elsevier 


\section{Introduction}

Material layers within a macroscopic continuum play an important role in numerous engineering applications. Examples include adhesive bonding layers, laminated composite structures, geomaterials and masonry structures. The material layer is generally weaker than the surrounding bulk causing the deformation to localise in the layer. The ability to model the response of the material layer is therefore critical in order to determine the behaviour of the overall continuum. The response of the material layer is dependent upon its microstructure and, in particular, heterogeneities. Microscopic heterogeneities include voids, inclusions and micro-cracks. Homogenisation, as pioneered by Hill [2, 3], provides a consistent methodology to link the macroscopic and microscopic scales and forms the basis for computational micro-to-macro transitions [4-14]. The key contribution of this work is to significantly extend the two-dimensional computational micro-to-macro transition approach for material layers proposed by Hirschberger et al. [15] to consistently account for in-plane stretch by endowing the macroscopic interface with its own energetic structure in the spirit of Gurtin and Murdoch [1]. The importance and relevance of considering materials layers using a multi-scale paradigm was commented upon in a recent overview paper by Geers et al. [16].

The kinematic behaviour of a material layer can be approximated as that of an incoherent interface. Thereby the displacement field exhibits a discontinuity across the interface.

The vast majority of formulations for material layers do not permit the layer to undergo in-plane stretch. The constitutive response of the material layer itself can be approximated using a cohesive traction-separation law. The traction-separation law relates the discontinuity in the macroscopic displacement field across the material layer (interface) to an interface traction [see e.g. 17-19]. The traction-separation law is, therefore, a constitutive model relating kinematic and kinetic quantities. The point of departure in Hirschberger et al. [15] was to circumvent the constitutive assumptions of the traction-separation law by using micro-to-macro transitions. The in-plane stretch of the interface was not, however, accounted for. Related works include [20-24].

An alternative view of an (incoherent) interface, or a surface, is obtained by endowing the interface with its own energetic structure in the form of a Helmholtz energy, as proposed in the seminal work of Gurtin and Murdoch [1] [see also 25-32]. The interface is thereby elevated to the same status as 
the bulk and obeys its own set of governing equations and constitutive relations. The governing equations for the interface are coupled to the response of the bulk. Kinematic quantities on the interface describe the deformation state. Constitutive relations for the interface, arising from consistent thermodynamic restrictions, relate kinematic and kinetic interface quantities. The objective of this work is to extend such a theory to account for micro-to-macro transitions, and thereby circumvent classical constitutive relations.

Related works includes those by Geers et al. [33] and Coenen et al. [34] on the response of macroscopic thin sheets with heterogeneous microstructure using second-order computational homogenisation schemes [35-38]. The macroscopic response is described by a fourth-order shell theory. A second-order computational homogenisation scheme is required to transfer the higher-order macroscopic kinematics to the microscopic problem. As in the theory presented here for material layers, measures of the surface kinematics and kinetics are required. Furthermore, the thickness of the shell governs the height of the representative volume element (RVE) at the microscopic scale. The same assumption regarding the thickness of the material layer is made here. A key distinction between these approaches and the one presented here is that a first-order homogenisation theory suffices for material layers where the macroscopic response is based upon the original theory of Gurtin and Murdoch [1].

Interface homogeneities have also been considered in the context of contact problems [see e.g. 39, 40].

In summary, the key objectives and contributions of this work are as follows:

- to state the equations governing an energetic interface accounting for in-plane stretch at the macroscopic scale;

- to consistently transfer the macroscopic kinematic interface data to the underlying microscopic problem and to determine the corresponding restrictions on the microscopic motion;

- to determine the macroscopic kinetic quantities and the interface tangent modulus from the averages of various microscopic fields;

- to solve the three-dimensional, nonlinear microscopic problem using the finite element method; 
- to elucidate the key features of the theory using a series of representative numerical examples involving heterogeneous microstructures.

This manuscript is organised as follows. The notation and certain key concepts are briefly introduced. The equations governing the response of the macroscopic bulk and the interface are then given in Section 2. This requires the introduction of various surface operators and geometric concepts. The microscopic problem is then introduced in Section 3. The Hill-type averaging condition is then employed to relate micro- and macroscopic quantities by exploiting the invariance of the variational work. The focus of this section is on the link between the two scales for a macroscopic point on the interface. Details of the numerical approximation using the finite element method are briefly given in Section 4. Thereafter, a series of representative numerical examples serve to elucidate the theory. We conclude and discuss further work in Section 6.

\section{Notation}

The purpose of this preliminary section is to summarise certain key concepts in nonlinear continuum mechanics and to introduce the notation adopted here. Detailed expositions on nonlinear continuum mechanics can be found in [41-44], among others.

Direct notation is used throughout. In addition, occasional use is made of index notation. Upper and lower case Roman indices refer to tensor components defined on the material and spatial configurations, respectively. The scalar product of two first-order tensors $\boldsymbol{a}$ and $\boldsymbol{b}$ is denoted $\boldsymbol{a} \cdot \boldsymbol{b}=a_{i} b_{i}$. The scalar product of two second-order tensors $\boldsymbol{A}$ and $\boldsymbol{B}$ is denoted $\boldsymbol{A}$ : $\boldsymbol{B}=A_{i j} B_{i j}$. The action of a second-order tensor $\boldsymbol{A}$ on a first-order tensor $\boldsymbol{b}$ is represented as $\boldsymbol{A} \cdot \boldsymbol{b}$, and in index notation as $A_{i j} b_{j}$. The action of a third-order tensor $\boldsymbol{\Lambda}$ on a first-order tensor $\boldsymbol{b}$ is represented as $\boldsymbol{\Lambda} \cdot \boldsymbol{b}$ with components $\Lambda_{i j k} b_{k}$. The tensor product $\boldsymbol{a} \otimes \boldsymbol{b}$ of two first-order tensors $\boldsymbol{a}$ and $\boldsymbol{b}$ is a second-order tensor $\boldsymbol{D}$ defined by the relation $[\boldsymbol{a} \otimes \boldsymbol{b}] \cdot \boldsymbol{c}=$ $[\boldsymbol{b} \cdot \boldsymbol{c}] \boldsymbol{a} \forall$ vectors $\boldsymbol{c}$ and is expressed in index notation as $D_{i j}=a_{i} b_{j}$. The non-standard tensor product of a second-order tensor $\boldsymbol{A}$ and a first-order tensor $\boldsymbol{b}$, denoted $\boldsymbol{A} \otimes \boldsymbol{b}$, is a third-order tensor with components $A_{i j} b_{k}$. The non-standard tensor product of a first-order tensor $\boldsymbol{a}$ and a second-order tensor $\boldsymbol{B}$, denoted $\boldsymbol{a} \otimes \boldsymbol{B}$, is a third-order tensor with components $a_{i} B_{j k}$. The non-standard tensor product of a first-order tensor $\boldsymbol{a}$ and a secondorder tensor $\boldsymbol{B}$, denoted $\boldsymbol{a} \bar{\otimes} \boldsymbol{B}$, is a third-order tensor with components 
$[\boldsymbol{a} \bar{\otimes} \boldsymbol{B}]_{i j k}=a_{j} B_{i k}$. Finally, the non-standard tensor product of a secondorder tensor $\boldsymbol{A}$ with another second-order tensor $\boldsymbol{B}$, denoted $\boldsymbol{A} \bar{\otimes} \boldsymbol{B}$, is a fourth-order tensor with components $[\boldsymbol{A} \bar{\otimes} \boldsymbol{B}]_{i j k l}=A_{i k} B_{j l}$.

Quantities defined on the macroscopic scale are differentiated from those on the microscopic scale by an accent placed above the symbol. That is, $\{\vec{\bullet}\},\{\hat{\bullet}\},\{\widetilde{\bullet}\}$ and $\{\vec{\bullet}\}$ refer to variables defined at the macroscopic scale. The notation $\{\overline{\boldsymbol{\bullet}}\}$ does not denote closure here.

Matrices and vectors are distinguished from second- and first-order tensors via upright font and are indicated in bold.

We distinguish indices unrelated to the number of space dimensions from those related to the number of space dimensions by using upright font for the former.

\section{The macroscopic problem}

The kinematics of a macroscopic continuum containing an energetic interface are presented in Section 2.1. This is followed by a discussion on superficial tensors. Various important integral relations are then given. With this preliminary material at hand, the macroscopic boundary value problem is stated in Section 2.4.

\subsection{Kinematics}

We denote by the open set $\overline{\mathcal{B}}_{0} \subset \mathbb{R}^{3}$ the material placement of a macroscopic continuum body, as depicted in Fig. 1. The external surface of the body $\overline{\mathcal{B}}_{0}$ is denoted $\partial \overline{\mathcal{B}}_{0}$ with unit outward normal $\overline{\boldsymbol{N}}$. Material particles in the material configuration are labelled $\overline{\boldsymbol{X}} \in \overline{\mathcal{B}}_{0}$. The body $\overline{\mathcal{B}}_{0}$ is partitioned into two distinct subdomains, $\overline{\mathcal{B}}_{0}^{-}$and $\overline{\mathcal{B}}_{0}^{+}$, by a two-sided interface $\overline{\mathcal{I}}_{0}$. The actual thickness of the interface in the material configuration is denoted $\bar{h}_{0}$. Material particles on the interface in the material configuration are denoted $\widehat{\boldsymbol{X}} \in \overline{\mathcal{I}}_{0}$. The boundaries of the subdomains $\overline{\mathcal{B}}_{0}^{-}$and $\overline{\mathcal{B}}_{0}^{+}$are denoted $\partial \overline{\mathcal{B}}_{0}^{-}$ and $\partial \overline{\mathcal{B}}_{0}^{+}$, respectively. The boundary of $\overline{\mathcal{I}}_{0}$, a curve, is denoted $\partial \overline{\mathcal{I}}_{0}$ with unit outward normal $\widehat{\boldsymbol{M}}$. The vector $\widehat{\boldsymbol{M}}$ is clearly tangent to the interface $\overline{\mathcal{I}}_{0}$. The two sides of the interface $\overline{\mathcal{I}}_{0}$ are denoted $\overline{\mathcal{I}}_{0}^{-}:=\partial \overline{\mathcal{B}}_{0}^{-} \cap \overline{\mathcal{I}}_{0}$ and $\overline{\mathcal{I}}_{0}^{+}:=\partial \overline{\mathcal{B}}_{0}^{+} \cap \overline{\mathcal{I}}_{0}$. The outward unit normals to the surfaces $\overline{\mathcal{I}}_{0}^{+}$and $\overline{\mathcal{I}}_{0}^{-}$are denoted $\overline{\boldsymbol{M}}^{+}$and $\overline{\boldsymbol{M}}^{-}$, respectively, with $\overline{\boldsymbol{M}}^{+}=-\overline{\boldsymbol{M}}^{-}$. Furthermore, the normal $\overline{\boldsymbol{M}}$ is defined by $\overline{\boldsymbol{M}}:=\overline{\boldsymbol{M}}^{-}$.

Let $\overline{\mathbb{T}}=[0, T] \subset \mathbb{R}_{+}$denote the time domain. Here time simply provides a history parameter to order the sequence of events and quasi-static condi- 


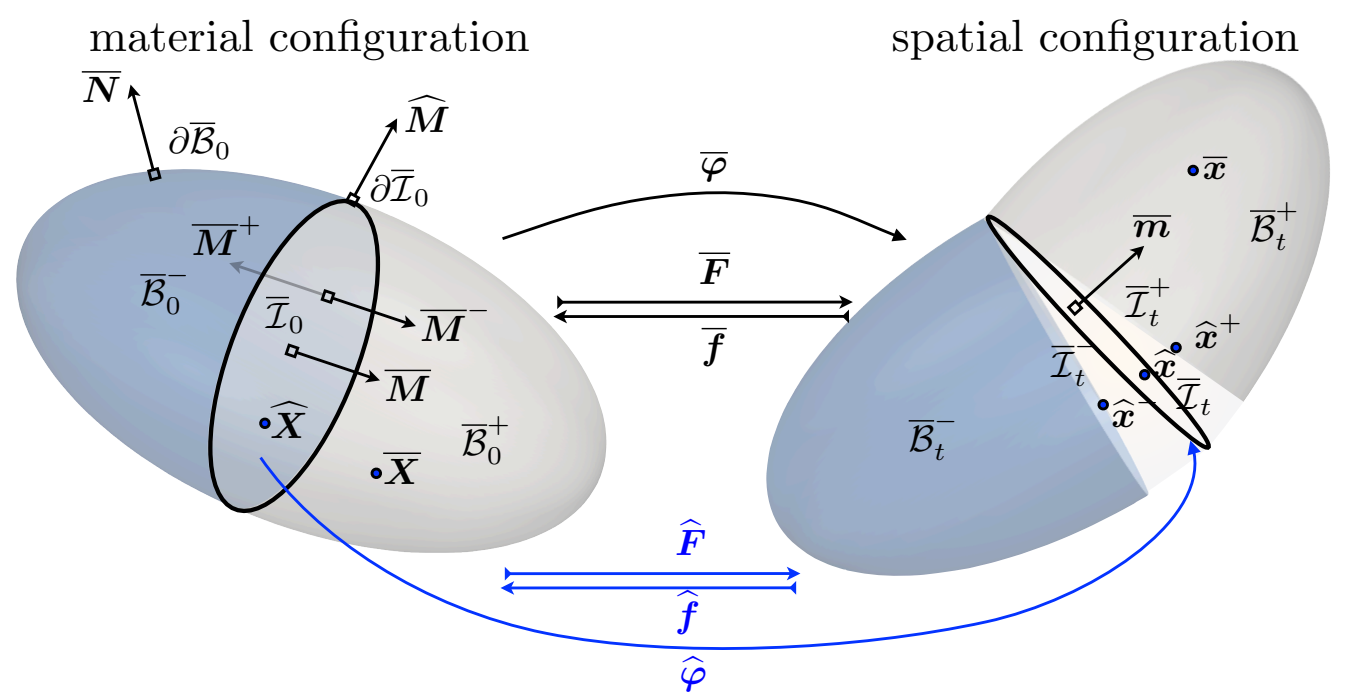

Figure 1: The motion $\bar{\varphi}$ of the macroscopic body from the material configuration $\overline{\mathcal{B}}_{0}$ to the spatial configuration $\overline{\mathcal{B}}_{t}$.

tions are assumed henceforth. ${ }^{1}$ We denote by the map $\bar{\varphi}: \overline{\mathcal{B}}_{0} \times \overline{\mathbb{T}} \rightarrow \mathbb{R}^{3}$, a motion of the macroscopic material placement $\overline{\mathcal{B}}_{0}$ for a time $t \in \overline{\mathbb{T}}$. The restriction of the motion $\bar{\varphi}$ to the interface $\overline{\mathcal{I}}_{0}$ is denoted $\widehat{\varphi}$. The current placement of the macroscopic body at a time $t$ associated with the motion $\bar{\varphi}$ is denoted $\overline{\mathcal{B}}_{t}=\overline{\boldsymbol{\varphi}}\left(\overline{\mathcal{B}}_{0}(\overline{\boldsymbol{X}}), t\right)$ with particles designated as $\overline{\boldsymbol{x}}=\overline{\boldsymbol{\varphi}}(\overline{\boldsymbol{X}}, t) \in \overline{\mathcal{B}}_{t}{ }^{2}$ The interface in the spatial configuration $\overline{\mathcal{I}}_{t}$ is defined as the surface midway between the negative and positive faces, $\overline{\mathcal{I}}_{t}^{-}$and $\overline{\mathcal{I}}_{t}^{+}$, respectively. That this is indeed a correct definition of the interface in the spatial configuration is discussed in [19]. Particles on the interface in the spatial configuration are denoted $\widehat{\boldsymbol{x}} \in \overline{\mathcal{I}}_{t}$. Particles on the negative and positive sides of the interface in the spatial configuration are denoted $\widehat{\boldsymbol{x}}^{-} \in \overline{\mathcal{I}}_{t}^{-}$and $\widehat{\boldsymbol{x}}^{+} \in \overline{\mathcal{I}}_{t}^{+}$, respectively.

The macroscopic, invertible, linear tangent map $\overline{\boldsymbol{F}}: T \overline{\mathcal{B}}_{0} \rightarrow T \overline{\mathcal{B}}_{t}$ (that is, the deformation gradient) maps a line element $\mathrm{d} \overline{\boldsymbol{X}} \in T \overline{\mathcal{B}}_{0}$ in the material configuration to a line element $\mathrm{d} \overline{\boldsymbol{x}} \in T \overline{\mathcal{B}}_{t}$ in the spatial configuration and is defined as the derivative of the macroscopic motion $\bar{\varphi}$ with respect to the material placement; that is,

$$
\overline{\boldsymbol{F}}(\overline{\boldsymbol{X}}, t)=\overline{\operatorname{Grad}} \overline{\boldsymbol{\varphi}}(\overline{\boldsymbol{X}}, t),
$$

\footnotetext{
${ }^{1}$ Computational temporal homogenisation is thus not considered.

${ }^{2}$ Here and henceforth, the subscripts $t$ and 0 shall designate spatial and material quantities, respectively, unless specified otherwise.
} 
where $\overline{\operatorname{Grad}}\{\bar{\bullet}\}:=\partial\{\bar{\bullet}\} / \partial \overline{\boldsymbol{X}}$ is the gradient operator with respect to the macroscopic material placement. The notation $T\{\bullet\}$ denotes the tangent space to $\{\bullet\}$. The Jacobian determinant of the macroscopic deformation gradient is denoted $\bar{J}(\overline{\boldsymbol{X}}, t):=\overline{\operatorname{det}}(\overline{\boldsymbol{F}}(\overline{\boldsymbol{X}}, t))>0$, where

$$
\overline{\operatorname{det}} \overline{\boldsymbol{F}}:=\frac{\left[\overline{\boldsymbol{F}} \cdot \overline{\boldsymbol{G}}_{1}\right] \cdot\left[\left[\overline{\boldsymbol{F}} \cdot \overline{\boldsymbol{G}}_{2}\right] \times\left[\overline{\boldsymbol{F}} \cdot \overline{\boldsymbol{G}}_{3}\right]\right]}{\overline{\boldsymbol{G}}_{1} \cdot\left[\overline{\boldsymbol{G}}_{2} \times \overline{\boldsymbol{G}}_{3}\right]},
$$

and $\overline{\boldsymbol{G}}_{I}(I=1,2,3)$ are the linearly independent material covariant vectors.

The inverse of the deformation gradient is denoted $\overline{\boldsymbol{f}}:=\overline{\boldsymbol{F}}^{-1}$ with an associated inverse Jacobian determinant $\bar{j}:=\overline{\operatorname{det}} \overline{\boldsymbol{f}}=1 / \bar{J}>0$, where

$$
\overline{\operatorname{det}} \overline{\boldsymbol{f}}:=\frac{\left[\overline{\boldsymbol{f}} \cdot \overline{\boldsymbol{g}}_{1}\right] \cdot\left[\left[\overline{\boldsymbol{f}} \cdot \overline{\boldsymbol{g}}_{2}\right] \times\left[\overline{\boldsymbol{f}} \cdot \overline{\boldsymbol{g}}_{3}\right]\right]}{\overline{\boldsymbol{g}}_{1} \cdot\left[\overline{\boldsymbol{g}}_{2} \times \overline{\boldsymbol{g}}_{3}\right]},
$$

and $\overline{\boldsymbol{g}}_{i}(i=1,2,3)$ are the linearly independent spatial covariant vectors. The condition on the motion that $\bar{J}(\overline{\boldsymbol{X}}, t)>0$ implies that the motion is invertible.

The non-invertible, linear interface tangent map $\widehat{\boldsymbol{F}}: T \overline{\mathcal{I}}_{0} \rightarrow T \overline{\mathcal{I}}_{t}$ (that is, the interface deformation gradient) maps tangential line elements on the interface in the material configuration $\mathrm{d} \widehat{\boldsymbol{X}} \in \overline{\mathcal{I}}_{0}$ to tangential line elements on the interface in the spatial configuration $\mathrm{d} \widehat{\boldsymbol{x}} \in \overline{\mathcal{I}}_{t}$. Although the linear surface tangent map $\widehat{\boldsymbol{F}}$ is non-invertible, due to rank deficiency, it possesses an inverse, denoted $\widehat{\boldsymbol{f}}$, in the following generalised sense:

$$
\widehat{\boldsymbol{F}} \cdot \widehat{\boldsymbol{f}}=\widehat{\boldsymbol{i}} \quad \text { and } \quad \widehat{\boldsymbol{f}} \cdot \widehat{\boldsymbol{F}}=\widehat{\boldsymbol{I}}
$$

where $\widehat{\boldsymbol{i}}$ and $\widehat{\boldsymbol{I}}$ are the mixed-variant interface unit tensors respectively defined by

$$
\widehat{\boldsymbol{i}}=\widehat{\mathbb{P}}_{t}:=\overline{\boldsymbol{i}}-\overline{\boldsymbol{m}} \otimes \overline{\boldsymbol{m}} \quad \text { and } \quad \widehat{\boldsymbol{I}}=\widehat{\mathbb{P}}_{0}:=\overline{\boldsymbol{I}}-\overline{\boldsymbol{M}} \otimes \overline{\boldsymbol{M}},
$$

and $\overline{\boldsymbol{i}}$ is the ordinary second-order mixed-variant unit tensor of the threedimensional embedding Euclidean space in the spatial configuration $\overline{\mathcal{B}}_{t}$. The second-order tensor $\overline{\boldsymbol{I}}$ is the ordinary mixed-variant unit tensor of the threedimensional embedding Euclidean space in the material configuration $\overline{\mathcal{B}}_{0}$.

The vector $\overline{\boldsymbol{m}}$ is the unit outward normal to $\overline{\mathcal{I}}_{t}$. It is clear that $\widehat{\boldsymbol{i}}$ and $\widehat{\boldsymbol{I}}$ are simply the spatial and material projections, denoted $\widehat{\mathbb{P}}_{t}$ and $\widehat{\mathbb{P}}_{0}$ respectively, onto the interfaces with normals $\overline{\boldsymbol{m}}$ and $\overline{\boldsymbol{M}}$, respectively. It is 
obvious that as the projection tensors depend on the interface normal, so do the interface unit tensors.

The interface deformation gradient $\widehat{\boldsymbol{F}}$ and its generalised inverse $\widehat{\boldsymbol{f}}$ are computed as the surface gradients of the interface deformation and its inverse, respectively. That is,

$$
\widehat{\boldsymbol{F}}:=\widehat{\operatorname{Grad}} \widehat{\boldsymbol{\varphi}}(\widehat{\boldsymbol{X}}, t) \quad \text { and } \quad \widehat{\boldsymbol{f}}:=\widehat{\operatorname{grad}} \widehat{\boldsymbol{\varphi}}^{-1}(\widehat{\boldsymbol{x}}, t),
$$

where

$$
\widehat{\operatorname{Grad}}\{\widehat{\bullet}\}:=\operatorname{Grad}\{\widehat{\bullet}\} \cdot \widehat{\boldsymbol{I}} \quad \text { and } \quad \widehat{\operatorname{grad}}\{\widehat{\bullet}\}:=\operatorname{grad}\{\widehat{\bullet}\} \cdot \widehat{\boldsymbol{i}}
$$

are simply the projections of the standard gradient operators onto the interface. The Jacobian determinant of the interface deformation gradient, denoted $\widehat{J}(\widehat{\boldsymbol{X}}, t):=\widehat{\operatorname{det}}(\widehat{\boldsymbol{F}}(\widehat{\boldsymbol{X}}, t))>0$, and its inverse $\widehat{j}(\widehat{\boldsymbol{x}}, t):=\widehat{\operatorname{det}}(\widehat{\boldsymbol{f}}(\widehat{\boldsymbol{x}}, t))>$ 0 , are defined by [see e.g. 31,45$]$ :

$$
\widehat{\operatorname{det}} \widehat{\boldsymbol{F}}:=\frac{\left|\left[\widehat{\boldsymbol{F}} \cdot \widehat{\boldsymbol{G}}_{1}\right] \times\left[\widehat{\boldsymbol{F}} \cdot \widehat{\boldsymbol{G}}_{2}\right]\right|}{\left|\widehat{\boldsymbol{G}}_{1} \times \widehat{\boldsymbol{G}}_{2}\right|} \quad \text { and } \quad \widehat{\operatorname{det} \widehat{\boldsymbol{f}}}:=\frac{\left|\left[\widehat{\boldsymbol{f}} \cdot \widehat{\boldsymbol{g}}_{1}\right] \times\left[\widehat{\boldsymbol{f}} \cdot \widehat{\boldsymbol{g}}_{2}\right]\right|}{\left|\widehat{\boldsymbol{g}}_{1} \times \widehat{\boldsymbol{g}}_{2}\right|}
$$

where $\widehat{\boldsymbol{G}}_{\alpha} \in T \overline{\mathcal{I}}_{0}(\alpha=1,2)$ and $\widehat{\boldsymbol{g}}_{\alpha} \in T \overline{\mathcal{I}}_{t}$ are the covariant interface basis vectors in the material and spatial configurations, respectively.

\subsection{Tensors and vectors}

A second-order tensor field on the interface $\overline{\mathcal{I}}_{0}$, arbitrarily denoted $\overline{\boldsymbol{S}}(\widehat{\boldsymbol{X}})$, is a linear transformation from the tangent space at $\widehat{\boldsymbol{X}}$ (the space of all vectors which are orthogonal to the interface normal vector $\overline{\boldsymbol{M}}$ ) to $\mathbb{R}^{3}$. This definition is made compatible with the standard definition of a second-order tensor as a linear transformation from $\mathbb{R}^{3}$ to $\mathbb{R}^{3}$ by requiring that the transformation of the interface normal vector by $\overline{\boldsymbol{S}}$ is precisely the zero vector [see e.g. 46]. Thus, the so-called superficial second-order tensor associated with $\overline{\boldsymbol{S}}$ is denoted by $\widehat{\boldsymbol{S}}: \mathbb{R}^{3} \rightarrow \mathbb{R}^{3}$ and satisfies the property that

$$
\widehat{S} \cdot \bar{M}=0 .
$$

One important example of a superficial second-order tensor is the material projection $\overline{\mathbb{P}}_{0}$ defined in Eq. (1) which maps an arbitrary vector in $\mathbb{R}^{3}$ tangent to the interface with normal $\overline{\boldsymbol{M}}$. 


\subsection{Jump and integral relations}

The jump and average operators for an arbitrary macroscopic field $\{\bar{\bullet}\}$ across the interface $\overline{\mathcal{I}}_{0}$ are respectively defined by

$$
\llbracket \bar{\bullet} \rrbracket:=\left.\{\bar{\bullet}\}\right|_{\overline{\mathcal{I}}_{0}^{+}}-\left.\{\bar{\bullet}\}\right|_{\overline{\mathcal{I}}_{0}^{-}} \quad \text { and } \quad\{\{\bar{\bullet}\}\}:=\frac{1}{2}\left[\left.\{\bar{\bullet}\}\right|_{\overline{\mathcal{I}}_{0}^{+}}+\left.\{\bullet\}\right|_{\overline{\mathcal{I}}_{0}^{-}}\right] \quad \text { on } \overline{\mathcal{I}}_{0} .
$$

Gauss's theorem, in its extended form, relates the material gradient (resp. divergence) of a quantity over the macroscopic material volume $\overline{\mathcal{B}}_{0}$, possibly containing an interface $\overline{\mathcal{I}}_{0}$, to the flux of the quantity over the external boundary $\partial \overline{\mathcal{B}}_{0}$, and the jump of the flux in the quantity over the interface $\overline{\mathcal{I}}_{0}$. Gauss's theorem for material scalars $\bar{a}_{0}$, and first- and secondorder tensors, collectively denoted $\overline{\boldsymbol{a}}_{0}$, is

$$
\begin{aligned}
\int_{\overline{\mathcal{B}}_{0}} \overline{\operatorname{Grad}} \bar{a}_{0} \mathrm{~d} V & =\int_{\partial \overline{\mathcal{B}}_{0}} \bar{a}_{0} \overline{\boldsymbol{N}} \mathrm{d} A-\int_{\overline{\mathcal{I}}_{0}} \llbracket \bar{a}_{0} \rrbracket \overline{\boldsymbol{M}} \mathrm{d} A, \\
\int_{\overline{\mathcal{B}}_{0}} \overline{\operatorname{Div}} \overline{\boldsymbol{a}}_{0} \mathrm{~d} V & =\int_{\partial \overline{\mathcal{B}}_{0}} \overline{\boldsymbol{a}}_{0} \cdot \overline{\boldsymbol{N}} \mathrm{d} A-\int_{\overline{\mathcal{I}}_{0}} \llbracket \overline{\boldsymbol{a}}_{0} \rrbracket \cdot \overline{\boldsymbol{M}} \mathrm{d} A .
\end{aligned}
$$

The following identity relates the jump of the scalar product of two quantities, denoted $\boldsymbol{a}$ and $\boldsymbol{b}$, to the products of their jumps and averages:

$$
\llbracket \boldsymbol{a} \cdot \boldsymbol{b} \rrbracket=\llbracket \boldsymbol{a} \rrbracket \cdot\{\{\boldsymbol{b}\}\}+\{\{\boldsymbol{a}\}\} \cdot \llbracket \boldsymbol{b} \rrbracket .
$$

\subsection{The macroscopic boundary value problem}

The equations governing the response of a nonlinear solid possessing an energetic material layer at the macroscopic scale are now summarised. For further information concerning energetic interfaces and surfaces the reader is referred to [1, 27-32] and the numerous references therein. The contributions by $\mathrm{Xu}$ and Needleman $[17,18]$ provide a detailed description of the mechanics of a cohesive interface.

The objective of a constitutive relation is to transform kinematic data concerning the deformation at a macroscopic material point into kinetic data representing the response of the continuum. In classical continuum theories the form of the constitutive relations in the bulk are represented by the Helmholtz energy $\bar{\psi}$. Similarly, the response of an energetic interface is characterised by a Helmholtz energy, denoted $\widehat{\psi}$, independent to that of the bulk.

The objective here is to sidestep these macroscopic constitutive approximations and to determine the constitutive response of the macroscopic 
material layer possessing an energetic interface directly from a simulation that captures the heterogeneous microstructure.

The external boundary of the macroscopic continuum $\partial \overline{\mathcal{B}}_{0}$ and the interface $\partial \overline{\mathcal{I}}_{0}$ are partitioned into Dirichlet and Neumann parts, denoted $\partial \overline{\mathcal{B}}_{0}^{\bar{\varphi}}$ and $\partial \overline{\mathcal{B}}_{0}^{\bar{t}}$, and $\partial \overline{\mathcal{I}}_{0}^{\widehat{\varphi}}$ and $\partial \overline{\mathcal{I}}_{0}^{\hat{t}}$ respectively, as follows:

$$
\begin{aligned}
\partial \overline{\mathcal{B}}_{0}=\partial \overline{\mathcal{B}}_{0}^{\bar{\varphi}} \cup \partial \overline{\mathcal{B}}_{0}^{\bar{t}} & \text { where } & \partial \overline{\mathcal{B}}_{0}^{\bar{\varphi}} \cap \partial \overline{\mathcal{B}}_{0}^{\bar{t}}=\emptyset, \\
\partial \overline{\mathcal{I}}_{0}=\partial \overline{\mathcal{I}}_{0}^{\widehat{\varphi}} \cup \partial \overline{\mathcal{I}}_{0}^{\widehat{t}} & \text { where } & \partial \overline{\mathcal{I}}_{0}^{\widehat{\varphi}} \cap \partial \overline{\mathcal{I}}_{0}^{\widehat{t}}=\emptyset .
\end{aligned}
$$

The strong form of the macroscopic boundary value problem, denoted $(\overline{\mathrm{S}})$, is that of finding the macroscopic motion $\bar{\varphi}: \overline{\mathcal{B}}_{0} \times \overline{\mathbb{T}} \rightarrow \mathbb{R}^{3}$ for a given body force $\overline{\boldsymbol{b}}_{0}$, prescribed boundary motions $\bar{\varphi}^{\mathrm{p}}: \partial \overline{\mathcal{B}}_{0}^{\bar{\varphi}} \rightarrow \mathbb{R}^{3}$ and $\widehat{\boldsymbol{\varphi}}^{\mathrm{p}}: \partial \overline{\mathcal{I}}_{0}^{\widehat{\varphi}} \rightarrow \mathbb{R}^{3}$ and prescribed boundary tractions $\overline{\boldsymbol{t}}_{0}^{\mathrm{p}}: \partial \overline{\mathcal{B}}_{0}^{\bar{t}} \rightarrow \mathbb{R}^{3}$ and $\widehat{\boldsymbol{t}}_{0}^{\mathrm{p}}: \partial \overline{\mathcal{I}}_{0}^{\widehat{t}} \rightarrow \mathbb{R}^{3}$, such that:

$$
(\overline{\mathrm{S}}) \begin{cases}\overline{\mathrm{Div}} \overline{\boldsymbol{P}}+\overline{\boldsymbol{b}}_{0} \doteq \mathbf{0}, & \text { in } \overline{\mathcal{B}}_{0}, \\ \overline{\boldsymbol{\varphi}} \doteq \overline{\boldsymbol{\varphi}}^{\mathrm{p}}, & \text { on } \partial \overline{\mathcal{B}}_{0}^{\bar{\varphi}} \\ \overline{\boldsymbol{P}} \cdot \overline{\boldsymbol{N}}:=\overline{\boldsymbol{t}}_{0} \doteq \overline{\boldsymbol{t}}_{0}^{\mathrm{p}}, & \text { on } \partial \overline{\mathcal{B}}_{0}^{\overline{\boldsymbol{t}}}, \\ \widehat{\operatorname{Div}} \widehat{\boldsymbol{P}}+\llbracket \overline{\boldsymbol{P}} \rrbracket \cdot \overline{\boldsymbol{M}} \doteq \mathbf{0}, & \text { on } \overline{\mathcal{I}}_{0}, \\ \widehat{\boldsymbol{\varphi}} \doteq \widehat{\boldsymbol{\varphi}}^{\mathrm{p}}, & \text { on } \partial \overline{\mathcal{I}}_{0}^{\bar{\varphi}} \\ \widehat{\boldsymbol{P}} \cdot \widehat{\boldsymbol{M}}:=\widehat{\boldsymbol{t}}_{0} \doteq \widehat{\boldsymbol{t}}_{0}^{\mathrm{p}} & \text { on } \partial \overline{\mathcal{I}}_{0}^{\widehat{\boldsymbol{t}}} \\ \{\overline{\boldsymbol{P}}\}\} \cdot \overline{\boldsymbol{M}}:=\widetilde{\boldsymbol{t}}_{0} & \text { on } \overline{\mathcal{I}}_{0} .\end{cases}
$$

The macroscopic Piola-Kirchhoff stress tensors in the bulk and on the interace are denoted $\overline{\boldsymbol{P}}$ and $\widehat{\boldsymbol{P}}$, respectively. The divergence operator on the interface is defined by

$$
\widehat{\operatorname{Div}}\{\widehat{\bullet}\}:=\widehat{\operatorname{Grad}}\{\widehat{\bullet}\}: \widehat{\boldsymbol{I}} .
$$

The statement of equilibrium in the bulk (5) and the associated Dirichlet and Neumann boundary conditions, (6) and (7) respectively, are standard. The statement of equilibrium on the interface $\overline{\mathcal{I}}_{0}$ is given by the generalised Young-Laplace equation (8). The divergence of the interface PiolaKirchhoff stress $\widehat{\boldsymbol{P}}$ is related to the jump in the macroscopic Piola-Kirchhoff stress $\llbracket \overline{\boldsymbol{P}} \rrbracket$ across the interface $\overline{\mathcal{I}}_{0}$. Dirichlet and Neumann boundary conditions on the boundary of the interface are given in Eq. (9)-(10). Finally, the interface traction $\widetilde{\boldsymbol{t}}_{0}$ is defined in Eq. (11). 
The strong form of the macroscopic governing equations considered by Hirschberger et al. [15] can be recovered by eliminating Eq. (9)-(10) from $(\overline{\mathrm{S}})$, and removing the term $\widehat{\operatorname{Div}} \widehat{\boldsymbol{P}}$ from Eq. (8).

To obtain the variational form of the macroscopic boundary value problem, denoted $(\overline{\mathrm{V}})$, from $(\overline{\mathrm{S}})$ we proceed formally. Taking the scalar product of Eq. (5) and (8) with an arbitrary motion in the bulk $\delta \overline{\boldsymbol{\varphi}} \in H_{0}^{1}\left(\overline{\mathcal{B}}_{0}\right)$ and on the interface $\delta \widehat{\boldsymbol{\varphi}} \in H_{0}^{1}\left(\overline{\mathcal{I}}_{0}\right)$, respectively, summing, integrating over the domain $\overline{\mathcal{B}}_{0}$, then integrating by parts and using Gauss's theorem (3), the relationship (4), the weak form of the Neumann boundary conditions given by Eq. (7) and (10) and the definition of the interface traction (11) yields:

$$
\begin{aligned}
\int_{\overline{\mathcal{B}}_{0}} & \overline{\boldsymbol{P}}: \delta \overline{\boldsymbol{F}} \mathrm{d} V+\int_{\overline{\mathcal{I}}_{0}} \widetilde{\boldsymbol{t}}_{0} \cdot \llbracket \delta \overline{\boldsymbol{\varphi}} \rrbracket \mathrm{d} A+\int_{\overline{\mathcal{I}}_{0}} \widehat{\boldsymbol{P}}: \delta \widehat{\boldsymbol{F}} \mathrm{d} A \\
& \left.+\int_{\overline{\mathcal{I}}_{0}}[\{\delta \delta \overline{\boldsymbol{\varphi}}\}\}-\delta \widehat{\boldsymbol{\varphi}}\right] \cdot[\llbracket \overline{\boldsymbol{P}} \rrbracket \cdot \overline{\boldsymbol{M}}] \mathrm{d} A \\
= & \int_{\overline{\mathcal{B}}_{0}} \overline{\boldsymbol{b}}_{0} \cdot \delta \overline{\boldsymbol{\varphi}} \mathrm{d} V+\int_{\partial \overline{\mathcal{B}}_{0}^{\bar{t}}} \overline{\boldsymbol{t}}_{0}^{\mathrm{p}} \cdot \delta \overline{\boldsymbol{\varphi}} \mathrm{d} A+\int_{\partial \overline{\mathcal{I}}_{0}^{\hat{t}}} \widehat{\boldsymbol{t}}_{0}^{\mathrm{p}} \cdot \delta \widehat{\boldsymbol{\varphi}} \mathrm{d} L,
\end{aligned}
$$

and assuming, as we did previously, that the average of the macroscopic motion on the interface is the interfacial motion, that is that $[\{\{\delta \overline{\boldsymbol{\varphi}}\}\}-\delta \widehat{\boldsymbol{\varphi}}]=$ $\mathbf{0} \forall \widehat{\boldsymbol{X}} \in \overline{\mathcal{I}}_{0}$, finally yields

$$
(\overline{\mathrm{V}})\left\{\begin{array}{c}
\int_{\overline{\mathcal{B}}_{0}} \overline{\boldsymbol{P}}: \delta \overline{\boldsymbol{F}} \mathrm{d} V+\int_{\overline{\mathcal{I}}_{0}} \widetilde{\boldsymbol{t}}_{0} \cdot \llbracket \delta \overline{\boldsymbol{\varphi}} \rrbracket \mathrm{d} A+\int_{\overline{\mathcal{I}}_{0}} \widehat{\boldsymbol{P}}: \delta \widehat{\boldsymbol{F}} \mathrm{d} A \\
=\int_{\overline{\mathcal{B}}_{0}} \overline{\boldsymbol{b}}_{0} \cdot \delta \overline{\boldsymbol{\varphi}} \mathrm{d} V+\int_{\partial \overline{\mathcal{B}}_{0}^{\bar{t}}} \overline{\boldsymbol{t}}_{0}^{\mathrm{p}} \cdot \delta \overline{\boldsymbol{\varphi}} \mathrm{d} A+\int_{\partial \overline{\mathcal{I}}_{0}^{\hat{t}}} \widehat{\boldsymbol{t}}_{0}^{\mathrm{p}} \cdot \delta \widehat{\boldsymbol{\varphi}} \mathrm{d} L
\end{array}\right.
$$

for all $\delta \overline{\boldsymbol{\varphi}} \in H_{0}^{1}\left(\overline{\mathcal{B}}_{0}\right)$, where $\delta \widehat{\varphi} \equiv\{\{\delta \bar{\varphi}\}\}$.

The left-hand side of Eq. (12) represents the total variational work of the macroscopic continuum. Accordingly, the variational work (density) at the macroscopic scale $\delta \overline{\mathcal{W}}_{0}(\overline{\boldsymbol{X}})$ is defined by

$$
\delta \overline{\mathcal{W}}_{0}(\overline{\boldsymbol{X}})=\left\{\begin{array}{lll}
\overline{\boldsymbol{P}}: \delta \overline{\boldsymbol{F}} & {\left[\mathrm{N} / \mathrm{m}^{2}\right]} & \text { if } \overline{\boldsymbol{X}} \in \overline{\mathcal{B}}_{0} \\
\widetilde{\boldsymbol{t}}_{0} \cdot \llbracket \delta \overline{\boldsymbol{\varphi}} \rrbracket+\widehat{\boldsymbol{P}}: \delta \widehat{\boldsymbol{F}} & {[\mathrm{N} / \mathrm{m}]} & \text { if } \overline{\boldsymbol{X}} \in \overline{\mathcal{I}}_{0}
\end{array}\right.
$$

The relations given in Eq. (13) identify the work conjugate kinetic and kinematic quantities. In the theory developed by Hirschberger et al. [15] 
the macroscopic variational work on the interface did not contain the term $\widehat{\boldsymbol{P}}: \delta \widehat{\boldsymbol{F}}$ associated with the in-plane deformation of the interface.

We respectively denote by $\widehat{\boldsymbol{\Sigma}}=\left\{\widetilde{\boldsymbol{t}}_{0}, \widehat{\boldsymbol{P}}\right\}$ and $\widehat{\boldsymbol{\Xi}}=\{\llbracket \overline{\boldsymbol{\varphi}} \rrbracket, \widehat{\boldsymbol{F}}\}$ the set of macroscopic kinetic and kinematic variables defined on the interface $\overline{\mathcal{I}}_{0}$, henceforth termed the generalised macroscopic interface stress and deformation, respectively. The macroscopic variational work on the interface is thus given by

$$
\delta \overline{\mathcal{W}}_{0}(\overline{\boldsymbol{X}})=\widehat{\boldsymbol{\Sigma}}: \delta \widehat{\boldsymbol{\Xi}} \quad[\mathrm{N} / \mathrm{m}] \quad \text { for } \overline{\boldsymbol{X}} \in \overline{\mathcal{I}}_{0} .
$$

The rates of change of the generalised macroscopic interface stress and strain measures are related by the generalised macroscopic interface tangent modulus $\widehat{\mathcal{A}}$ as follows:

$$
\begin{aligned}
\dot{\hat{\boldsymbol{\Sigma}}} & =\widehat{\mathcal{A}}: \dot{\widehat{\boldsymbol{\Xi}}} \\
& =\left[\partial_{\widehat{\boldsymbol{\Xi}}} \widehat{\boldsymbol{\Sigma}}\right]: \dot{\hat{\boldsymbol{\Xi}}} \\
& =\left[\begin{array}{ll}
\partial_{\llbracket \bar{\varphi} \rrbracket} \widetilde{\boldsymbol{t}}_{0} & \partial_{\widehat{\boldsymbol{F}}} \widetilde{\boldsymbol{t}}_{0} \\
\partial_{\llbracket \bar{\varphi} \rrbracket} \widehat{\boldsymbol{P}} & \partial_{\widehat{\boldsymbol{F}}} \widehat{\boldsymbol{P}}
\end{array}\right]: \dot{\widehat{\boldsymbol{\Xi}}},
\end{aligned}
$$

where a superposed dot denotes the conventional material time derivative. The macroscopic tangent modulus $\widehat{\mathcal{A}}$ together with the macroscopic kinetic variable $\widehat{\Sigma}$ are computed from the response of the underlying microscopic problem.

\section{The microscopic problem}

The kinematics of the microscopic problem are now introduced. The microscopic boundary value problem is then described. The Hill-type averaging theorem is then exploited to link macro- and microscopic quantities, both in the bulk and on the interface.

\subsection{Kinematics}

In accordance with the assumption of scale separation, a microscopic RVE with material configuration $\mathcal{B}_{0} \subset \mathbb{R}^{3}$ is associated with each macroscopic material point $\widehat{\boldsymbol{X}}$ on the interface $\overline{\mathcal{I}}_{0}$, as depicted in Fig. 2. Material particles in $\mathcal{B}_{0}$ are labelled $\boldsymbol{X}$. Computational micro-to-macro transitions for material points within the bulk are also discussed briefly to introduce certain key concepts pertaining to material layers with energetic interfaces within a familiar setting. 


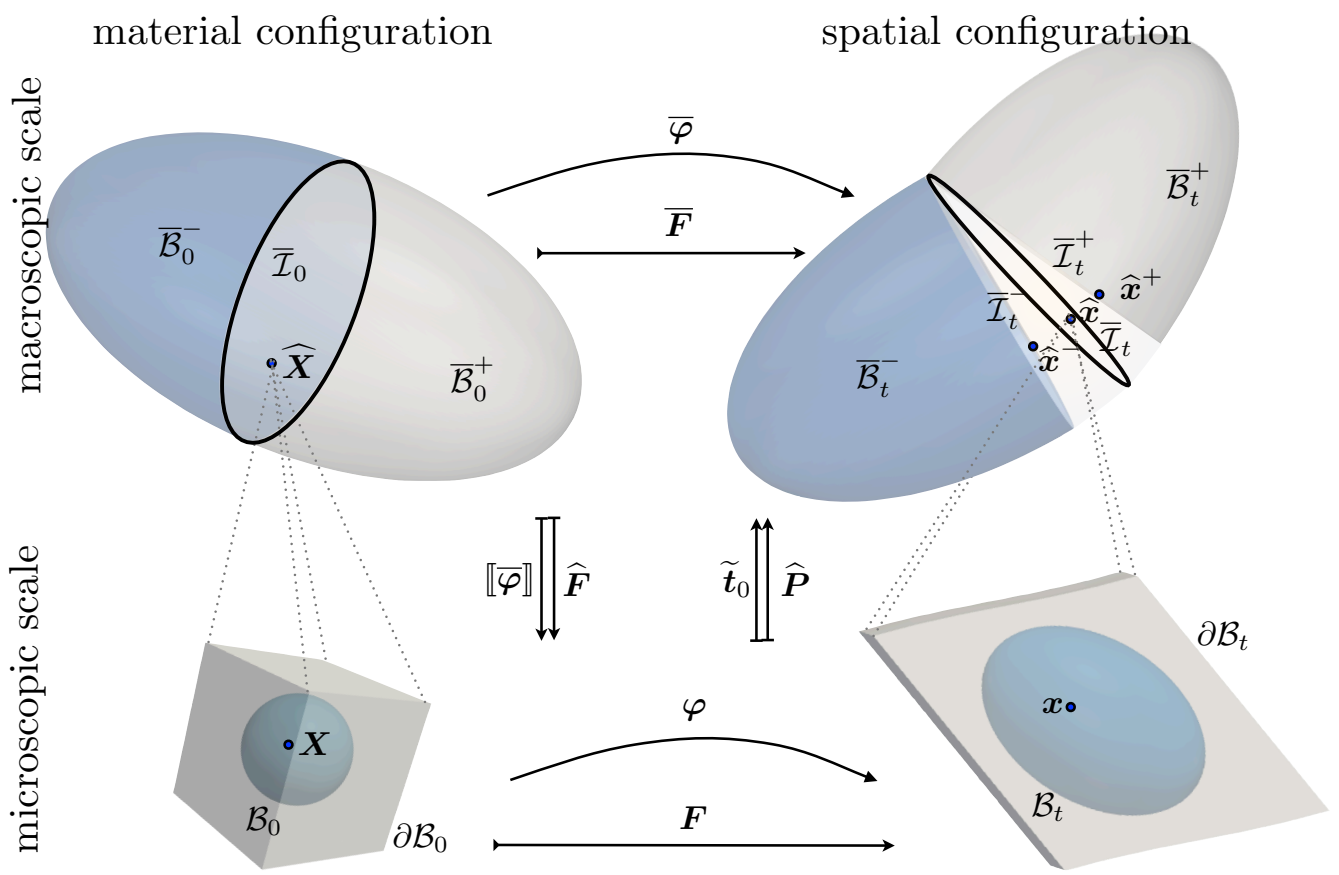

Figure 2: The motion $\varphi$ of the microscopic continuum body, associated with a macroscopic point $\widehat{\boldsymbol{X}}$ on the interface $\overline{\mathcal{I}}_{0}$, from the material configuration $\mathcal{B}_{0}$ to the spatial configuration $\mathcal{B}_{t}$.

It is important to note that a RVE is always a three-dimensional domain with volume $V_{0}>0$ even for macroscopic material particles on the interface $\overline{\mathcal{I}}_{0}$. Furthermore, care needs to be taken when transferring superficial, second-order tensors from the macroscopic interface to the underlying microscopic RVE. The extension of a macroscopic superficial, second-order tensor $\{\widehat{\bullet}\}$ to the three-dimensional RVE is denoted $\{\vec{\bullet}\}$ and will be elaborated upon further in the remark towards the end of Section 3.4.1.

The spatial placement of the RVE associated with the microscopic motion $\boldsymbol{\varphi}$ is denoted $\mathcal{B}_{t} \subset \mathbb{R}^{3}$ with particles labelled $\boldsymbol{x}=\boldsymbol{\varphi}(\boldsymbol{X}, t) \in \mathcal{B}_{t}$. The microscopic deformation gradient $\boldsymbol{F}: T \mathcal{B}_{0} \rightarrow T \mathcal{B}_{t}$ is defined as the derivative of the microscopic motion with respect to the material placement; that is,

$$
\boldsymbol{F}(\boldsymbol{X}, t):=\operatorname{Grad} \boldsymbol{\varphi}(\boldsymbol{X}, t),
$$

where $\operatorname{Grad}\{\bullet\}:=\partial\{\bullet\} / \partial \boldsymbol{X}$ is the gradient operator with respect to the microscopic material placement.

Consider first a RVE associated with a macroscopic material point $\overline{\boldsymbol{X}}$ 13 
within the bulk $\overline{\mathcal{B}}_{0}$ as depicted in Fig. 3 . For the sake of simplicity the RVE is assumed to be a cuboid with face dimensions $l_{0}^{I}$ where $I=1,2,3$. The boundary of the RVE is denoted $\partial \mathcal{B}_{0}$ with outward unit normal $\boldsymbol{N}$. The boundary can be further categorised into negative and positive surfaces depending on the orientation of the surface normal relative to the local coordinate system which is located at the centroid of the RVE with unit basis vectors $\boldsymbol{E}_{I}(I=1,2,3)$. Thus, $\partial \mathcal{B}_{0}=\partial \mathcal{B}_{0}^{-} \cup \partial \mathcal{B}_{0}^{+}$. Furthermore, there are three pairs of negative and positive faces, with the pairing based upon the normals being opposite.

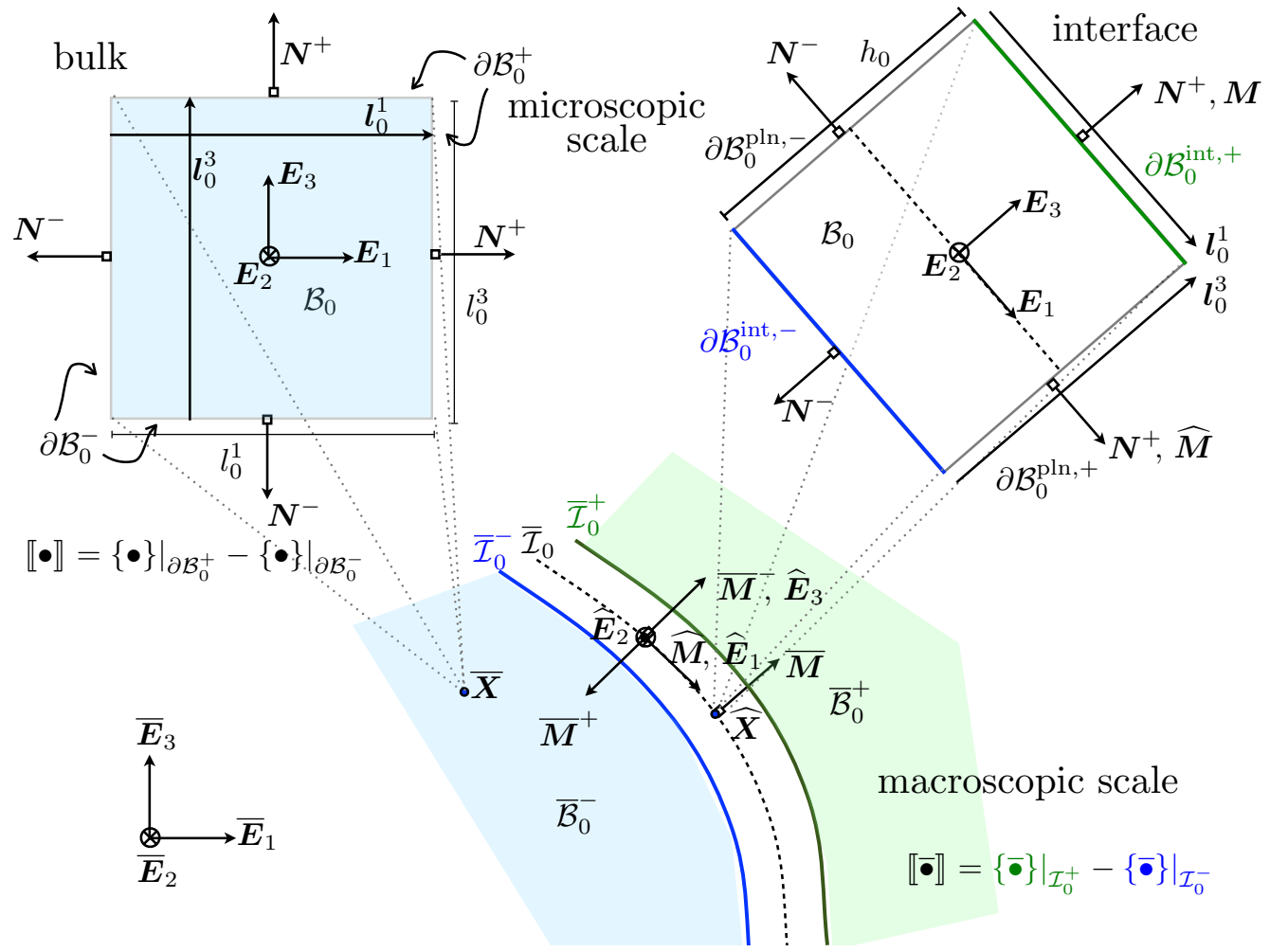

Figure 3: Typical RVEs corresponding to a material point in the bulk $\overline{\mathcal{B}}_{0}$ and on the interface $\overline{\mathcal{I}}_{0}$, respectively.

The vector between corresponding points on one of the three possible pairs of faces is defined by:

$$
\boldsymbol{l}_{0}^{I}:=\left.\boldsymbol{X}\right|_{\partial \mathcal{B}_{0}^{I,+}}-\left.\boldsymbol{X}\right|_{\partial \mathcal{B}_{0}^{I,-}} \quad \text { where } \quad I=1,2,3 .
$$

Next consider the case where the RVE corresponds to a macroscopic material point $\widehat{\boldsymbol{X}}$ on the interface $\overline{\mathcal{I}}_{0}$, as is also depicted in Fig. 3. A local 
coordinate system with basis vectors $\boldsymbol{E}_{I}$, where $I=1,2,3$, is chosen so as to coincide with the local coordinate system of the interface at the macroscopic scale. Specifically, $\widehat{\boldsymbol{E}}_{I}=\boldsymbol{E}_{I}(I=1,2,3)$. The initial height $h_{0}:=l_{0}^{3} \equiv \bar{h}_{0}$ of the RVE is fixed by the known initial thickness of the macroscopic material layer. Similar arguments are used when considering the computational homogenisation of macroscopic shells with an underlying heterogeneous microstructure [see e.g. 33, 34]. The faces of the RVE corresponding to the positive and negative surfaces of the interface, $\overline{\mathcal{I}}_{0}^{+}$and $\overline{\mathcal{I}}_{0}^{-}$respectively, are respectively denoted $\partial \mathcal{B}_{0}^{\text {int, }}+$ and $\partial \mathcal{B}_{0}^{\text {int, }}$. . The remaining pairs of opposite, in-plane faces are denoted $\partial \mathcal{B}_{0}^{\text {pln, }}$.

The jump and average operators between corresponding points on the opposite faces of the RVE for an arbitrary microscopic field $\{\bullet\}$ are respectively defined by

$$
\llbracket \bullet \rrbracket=\left.\{\bullet\}\right|_{\partial \mathcal{B}_{0}^{+}}-\left.\{\bullet\}\right|_{\partial \mathcal{B}_{0}^{-}} \quad \text { and } \quad\{\{\bullet\}\}=\frac{1}{2}\left[\left.\{\bullet\}\right|_{\partial \mathcal{B}_{0}^{+}}+\left.\{\bullet\}\right|_{\partial \mathcal{B}_{0}^{-}}\right] .
$$

The relation (4) between the jump and average of a quantity applies at both the macro- and microscopic scales. At the macroscopic scale, the jump is measured relative to the values on the positive and negative sides of the interface. At the microscopic scale, the jump is measured relative to the corresponding positive and negative faces of the RVE.

\subsection{Microscopic boundary value problem}

The microscopic continuum is subjected to applied loading transmitted from the macroscopic structure and obtained from the solution of the problem $(\overline{\mathrm{V}})$. The constitutive response of the heterogenous microstructure is obtained directly from a constitutive law following standard procedure [see e.g. 47]. The microstructure is thus fully resolved at this level of observation.

The characteristic in-plane lengths of the macro- and microscopic problems, denoted $\bar{l}_{0}$ and $l_{0}$ respectively, are assumed to be sufficiently separated such that $\bar{l}_{0} \gg l_{0}$. The separation of scales motivates the classical assumption that the response at the microscopic scale is not significantly influenced by volumetric forces.

The strong form of the microscopic boundary value problem $(\mathrm{S})$ is that of finding $\varphi: \mathcal{B}_{0} \times \mathbb{T} \rightarrow \mathbb{R}^{3}$ such that

$$
\text { (S) } \quad \operatorname{Div} \boldsymbol{P} \doteq \mathbf{0}, \quad \text { in } \mathcal{B}_{0},
$$

where $\boldsymbol{P}$ is the microscopic Piola-Kirchhoff stress. The time domain at the microscopic scale $\mathbb{T}$ is assumed to coincide with the macroscopic time 
domain $\overline{\mathbb{T}}$. The boundary conditions for the microscopic problem will be discussed in detail shortly.

The variational form of the microscopic boundary value problem (V) is obtained in a similar manner to the macroscopic problem $(\overline{\mathrm{V}})$ as

$$
\int_{\mathcal{B}_{0}} \boldsymbol{P}: \underbrace{\operatorname{Grad} \delta \boldsymbol{\varphi}}_{\delta \boldsymbol{F}} \mathrm{d} V=\int_{\partial \mathcal{B}_{0}} \boldsymbol{t}_{0} \cdot \delta \boldsymbol{\varphi} \mathrm{d} A,
$$

for all admissible, arbitrary motions $\delta \boldsymbol{\varphi} \in H_{0}^{1}\left(\mathcal{B}_{0}\right)$. The total variational work associated with the microscopic RVE is thus given by the left-hand side of Eq. (18).

\subsection{The Hill-type averaging condition}

The celebrated Hill-type averaging condition [2, 3] stipulates (variational) work equivalence between the macro- and microscopic scales. From the relations (13) at the macroscopic scale and (18) at the microscopic scale it follows that

$$
H:=\frac{\bar{d}_{0}(\overline{\boldsymbol{X}})}{V_{0}} \int_{\mathcal{B}_{0}} \boldsymbol{P}: \delta \boldsymbol{F} \mathrm{d} V-\delta \overline{\mathcal{W}}_{0}(\overline{\boldsymbol{X}}) \doteq 0
$$

where the scaling parameter $\bar{d}_{0}$ is defined by

$$
\bar{d}_{0}(\overline{\boldsymbol{X}}):=\left\{\begin{array}{ll}
1 & \text { if } \overline{\boldsymbol{X}} \in \overline{\mathcal{B}}_{0} \\
h_{0} & \text { if } \overline{\boldsymbol{X}} \in \overline{\mathcal{I}}_{0}
\end{array},\right.
$$

and is required to account for the dimensional mismatch between the twodimensional manifold embedded within three-dimensional space that is the interface and its microscopic representation as a three-dimensional object.

The volume averages of the following microscopic quantities will be required in the subsequent presentation:

$$
\begin{gathered}
\int_{\mathcal{B}_{0}} \boldsymbol{F} \mathrm{d} V=\int_{\partial \mathcal{B}_{0}} \boldsymbol{\varphi} \otimes \boldsymbol{N} \mathrm{d} A=\int_{\partial \mathcal{B}_{0}^{+}} \llbracket \boldsymbol{\varphi} \rrbracket \otimes \boldsymbol{N}^{+} \mathrm{d} A, \\
\int_{\mathcal{B}_{0}} \boldsymbol{P} \mathrm{d} V=\int_{\partial \mathcal{B}_{0}} \boldsymbol{t}_{0} \otimes \boldsymbol{X} \mathrm{d} A=\int_{\partial \mathcal{B}_{0}^{+}}\left[\llbracket \boldsymbol{X} \otimes \boldsymbol{P} \rrbracket \cdot \boldsymbol{N}^{+}\right]^{t} \mathrm{~d} A, \\
\int_{\mathcal{B}_{0}} \boldsymbol{P}: \boldsymbol{F} \mathrm{d} V=\int_{\partial \mathcal{B}_{0}} \boldsymbol{t}_{0} \cdot \boldsymbol{\varphi} \mathrm{d} A=\int_{\partial \mathcal{B}_{0}^{+}} \llbracket \boldsymbol{\varphi} \cdot \boldsymbol{P} \rrbracket \cdot \boldsymbol{N}^{+} \mathrm{d} A .
\end{gathered}
$$


The microscopic motion $\varphi$ is linked to the macroscopic deformation by the standard first-order ansatz:

$$
\boldsymbol{\varphi}(\boldsymbol{X}, t)=\overline{\mathcal{F}}(t) \cdot \boldsymbol{X}+\boldsymbol{w}(\boldsymbol{X}, t)
$$

where $\overline{\mathcal{F}}$ is a measure of the gradient of the effective macroscopic deformation field and $\boldsymbol{w}(\boldsymbol{X}, t)$ is the non-homogeneous fluctuation field. The form of the measure of the gradient of the effective macroscopic deformation field depends on the macroscopic kinematics. Where the macroscopic response is that of a classical continuum one obtains the well-known relationship $\overline{\mathcal{F}} \equiv \overline{\boldsymbol{F}}$ [see e.g. 12]. The form of $\overline{\mathcal{F}}$ corresponding to the kinematics associated with a macroscopic interface will be given in Section 3.4.1 [see 15, for inextensible interfaces]. Applying the definition of the microscopic deformation gradient $\boldsymbol{F}$ in Eq. (15) to the microscopic motion given in Eq. (23) yields:

$$
\boldsymbol{F}=\operatorname{Grad} \boldsymbol{\varphi} \equiv \overline{\mathcal{F}}+\operatorname{Grad} \boldsymbol{w}
$$

The variations of the motion $\boldsymbol{\varphi}$ and the deformation gradient $\boldsymbol{F}$ follow straightforwardly as

$$
\begin{aligned}
\delta \boldsymbol{\varphi} & =\delta \overline{\mathcal{F}} \cdot \boldsymbol{X}+\delta \boldsymbol{w}, \\
\delta \boldsymbol{F} & =\delta \overline{\mathcal{F}}+\operatorname{Grad} \delta \boldsymbol{w} .
\end{aligned}
$$

\subsubsection{Hill-type averaging condition for the bulk}

For a macroscopic point within the bulk $\overline{\mathcal{F}} \equiv \overline{\boldsymbol{F}}$. The Hill-type averaging condition (19) for the bulk is thus given by

$$
\begin{aligned}
0 \doteq H & =\frac{1}{V_{0}} \int_{\partial \mathcal{B}_{0}} \boldsymbol{t}_{0} \cdot \delta \boldsymbol{\varphi} \mathrm{d} A-\delta \overline{\boldsymbol{F}}: \overline{\boldsymbol{P}} \\
& =\delta \overline{\boldsymbol{F}}:\left[\frac{1}{V_{0}} \int_{\partial \mathcal{B}_{0}} \boldsymbol{t}_{0} \otimes \boldsymbol{X} \mathrm{d} A-\overline{\boldsymbol{P}}\right]+\frac{1}{V_{0}} \int_{\partial \mathcal{B}_{0}} \boldsymbol{t}_{0} \cdot \delta \boldsymbol{w} \mathrm{d} A \\
& \Longrightarrow \quad \overline{\boldsymbol{P}}=\frac{1}{V_{0}} \int_{\partial \mathcal{B}_{0}} \boldsymbol{t}_{0} \otimes \boldsymbol{X} \mathrm{d} A=\frac{1}{V_{0}} \int_{\mathcal{B}_{0}} \boldsymbol{P} \mathrm{d} V
\end{aligned}
$$

and thus

$$
\begin{aligned}
\Longrightarrow \quad 0 & \doteq \int_{\partial \mathcal{B}_{0}} \boldsymbol{t}_{0} \cdot \delta \boldsymbol{w} \mathrm{d} A \\
& =\int_{\partial \mathcal{B}_{0}^{+}}[\llbracket \delta \boldsymbol{w} \rrbracket \cdot\{\{\boldsymbol{P}\}\}+\{\{\delta \boldsymbol{w}\}\} \cdot \llbracket \boldsymbol{P} \rrbracket] \cdot \boldsymbol{N}^{+} \mathrm{d} A .
\end{aligned}
$$


The Hill-type averaging condition is satisfied by imposing one of the following three constraints on the fluctuation field:

(i) $\underbrace{\delta \boldsymbol{w}=\mathbf{0} \text { in } \mathcal{B}_{0}}_{\text {Taylor } / \text { Voigt }}$, (ii) $\underbrace{\delta \boldsymbol{w}=\mathbf{0} \text { on } \partial \mathcal{B}_{0}}_{\text {kinematic }}$, (iii) $\underbrace{\llbracket \delta \boldsymbol{w} \rrbracket=\mathbf{0} \text { on } \partial \mathcal{B}_{0}^{+}}_{\text {periodic fluctuations }}$.

If periodic fluctuations are chosen as specified in Eq. $(27)_{3}$, then $\{\{\delta \boldsymbol{w}\}\}=$ $\delta \boldsymbol{w}$ and the Hill-type averaging condition (19) is satisfied if

$$
\llbracket \boldsymbol{P} \rrbracket \cdot \boldsymbol{N}^{+}=\mathbf{0} \quad \text { and consequently } \quad\left\{\left\{\boldsymbol{t}_{0}\right\}\right\}=\mathbf{0} .
$$

The relation (25) identifies the macroscopic Piola-Kirchhoff stress $\overline{\boldsymbol{P}}$ as the microscopic volume average of the microscopic Piola-Kirchhoff stress $\boldsymbol{P}$. As is standard, we relate the microscopic and macroscopic deformation gradients as follows:

$$
\overline{\boldsymbol{F}}=\frac{1}{V_{0}} \int_{\mathcal{B}_{0}} \boldsymbol{F} \mathrm{d} V
$$

REMARK. Assuming periodic fluctuations, the following expression for the macroscopic Piola-Kirchhoff $\overline{\boldsymbol{P}}$ stress is convenient for the subsequent numerical implementation as only the tractions on the positive faces of the RVE need be computed [48]:

$$
\overline{\boldsymbol{P}}=\frac{1}{V_{0}} \int_{\partial \mathcal{B}_{0}^{+}} \boldsymbol{t}_{0}^{+} \otimes \llbracket \boldsymbol{X} \rrbracket \mathrm{d} A=\frac{1}{V_{0}} \sum_{\alpha=1}^{3} \underbrace{\int_{\partial \mathcal{B}_{0}^{\alpha,+}} \boldsymbol{t}_{0}^{+} \mathrm{d} A}_{\boldsymbol{f}_{0}^{\alpha}} \otimes \boldsymbol{l}_{0}^{\alpha},
$$

where the stress resultant on the positive face $\alpha$ is denoted $\boldsymbol{f}_{0}^{\alpha}$.

\subsection{The Hill-type averaging condition for the interface}

\subsubsection{Kinematics constraints}

The upper and lower boundaries of the problem on the microscopic level $\partial \mathcal{B}_{0}^{\text {int, }} \pm$ are defined to coincide with the positive and negative sides of the macroscopic interface $\overline{\mathcal{I}}_{0}^{ \pm}$. As such $\partial \mathcal{B}_{0}^{\text {int, } \pm}$ are subject to Dirichlet boundary conditions arising directly from the macroscopic problem and the nonhomogeneous fluctuation field $\boldsymbol{w}$ is, by definition, zero here. Motivated by the choice of periodic boundary conditions for the bulk in Section 3.3.1 and 
in the work of Hirschberger et al. [15], we assume a priori periodic fluctuations on the remaining in-plane faces $\partial \mathcal{B}_{0}^{\text {pln }}$. It will be shown shortly that these assumptions fulfil the Hill-type averaging condition.

The macroscopically imposed Dirichlet boundary conditions on $\partial \mathcal{B}_{0}^{\text {int, }} \pm$ are thus

$$
\boldsymbol{\varphi}= \pm \frac{1}{2} \llbracket \overline{\boldsymbol{\varphi}} \rrbracket+\overrightarrow{\boldsymbol{F}} \cdot \boldsymbol{X} \quad \text { on } \partial \mathcal{B}_{0}^{\mathrm{int}, \pm}
$$

and hence

$$
\llbracket \varphi \rrbracket=\llbracket \overline{\boldsymbol{\varphi}} \rrbracket+\overrightarrow{\boldsymbol{F}} \cdot \llbracket \boldsymbol{X} \rrbracket \quad \text { on } \partial \mathcal{B}_{0}^{\text {int, }+}
$$

as indicated in Fig. 4. The macroscopic interface deformation gradient $\overrightarrow{\boldsymbol{F}}$ is the extension of the superficial, second-order tensor $\widehat{\boldsymbol{F}}$ to $\mathbb{R}^{3}$. The boundary conditions (31) can be interpreted as follows. The jump in the macroscopic motion $\llbracket \bar{\varphi} \rrbracket$ is split equally between the faces $\partial \mathcal{B}_{0}^{\text {int, }} \pm$. Additionally the faces are allowed to deform due the applied macroscopic interface deformation gradient $\overrightarrow{\boldsymbol{F}}$. It is clear from (31) that the faces $\partial \mathcal{B}_{0}^{\text {int, }}{ }^{ \pm}$must remain parallel. On the remaining in-plane faces $\partial \mathcal{B}_{0}^{\text {pln }}$ the motion and the jump thereof are given by

$$
\begin{aligned}
\boldsymbol{\varphi} & =\frac{\boldsymbol{X} \cdot \boldsymbol{M}}{h_{0}} \llbracket \overline{\boldsymbol{\varphi}} \rrbracket+\overrightarrow{\boldsymbol{F}} \cdot \boldsymbol{X}+\boldsymbol{w} & & \text { on } \partial \mathcal{B}_{0}^{\mathrm{pln}}, \\
\llbracket \boldsymbol{\varphi} \rrbracket & =\overrightarrow{\boldsymbol{F}} \cdot \llbracket \boldsymbol{X} \rrbracket & & \text { on } \partial \mathcal{B}_{0}^{\mathrm{pln},+},
\end{aligned}
$$

where $\boldsymbol{X} \cdot \boldsymbol{M}=[\boldsymbol{X}]_{3}$. Thus, the contribution of the macroscopic displacement jump $\llbracket \overline{\boldsymbol{\varphi}} \rrbracket$ to $\boldsymbol{\varphi}$ varies linearly with vertical position on the faces $\partial \mathcal{B}_{0}^{\text {pln }}$.

From the Dirichlet boundary conditions on $\partial \mathcal{B}_{0}^{\text {int, }} \pm$ given in Eq. (31)(32), and assuming the motion on $\partial \mathcal{B}_{0}^{\text {pln }}$ to conform to the constraints given in Eq. (33)-(34), the measure of the effective macroscopic deformation gradient $\overline{\mathcal{F}}$ is related to the macroscopic kinematic quantities $\widehat{\boldsymbol{F}}$ and $\llbracket \overline{\boldsymbol{\varphi}} \rrbracket$ as 


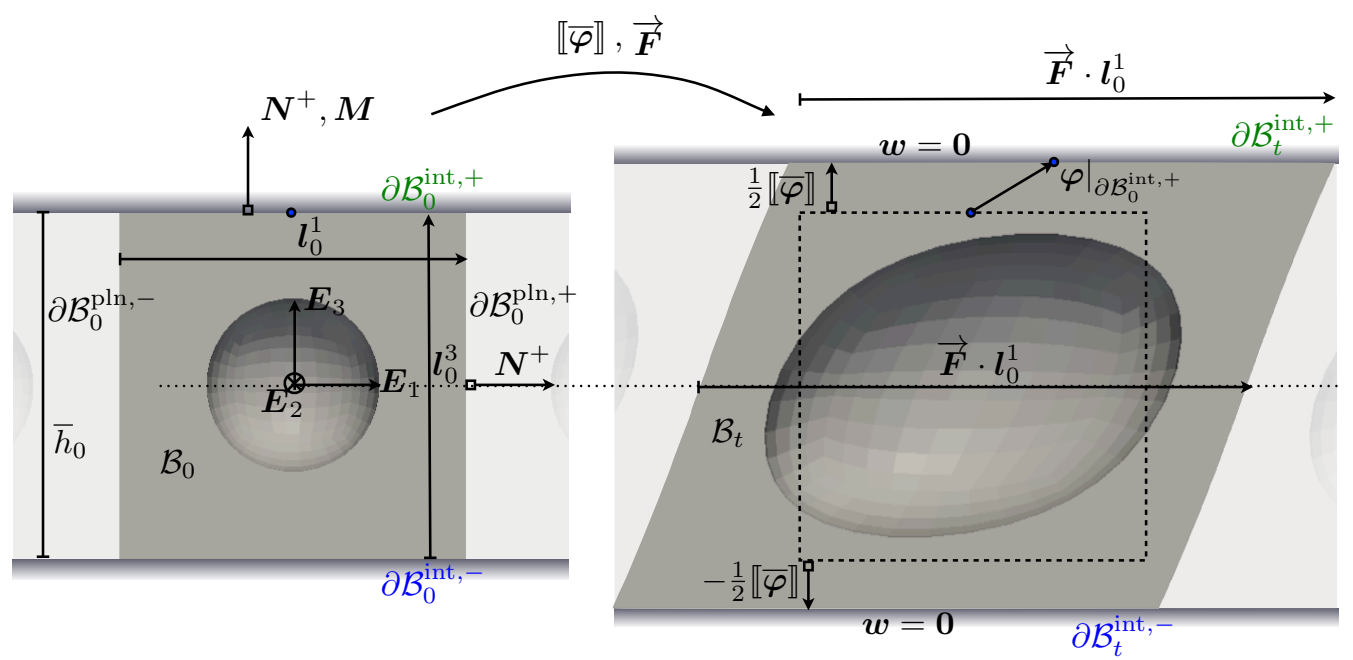

Figure 4: Schematic of the boundary conditions on the RVE arising from the macroscopic interface's kinematics and the assumption of in-plane periodicity.

follows:

$$
\begin{aligned}
& \overline{\mathcal{F}}=\frac{1}{V_{0}} \int_{\mathcal{B}_{0}} \boldsymbol{F} \mathrm{d} V \\
& =\frac{1}{V_{0}} \int_{\partial \mathcal{B}_{0}} \boldsymbol{\varphi} \otimes \boldsymbol{N} \mathrm{d} A \\
& =\frac{1}{V_{0}}\left[\int_{\partial \mathcal{B}_{0}^{\mathrm{pln},+}} \llbracket \boldsymbol{\varphi} \rrbracket \otimes \boldsymbol{N}^{+} \mathrm{d} A+\int_{\partial \mathcal{B}_{0}^{\mathrm{int},+}} \llbracket \boldsymbol{\varphi} \rrbracket \otimes \boldsymbol{N}^{+} \mathrm{d} A\right] \\
& =\frac{1}{V_{0}}\left[\int_{\partial \mathcal{B}_{0}^{\mathrm{pln},+}}[\overrightarrow{\boldsymbol{F}} \cdot \llbracket \boldsymbol{X} \rrbracket] \otimes \boldsymbol{N}^{+} \mathrm{d} A+\int_{\partial \mathcal{B}_{0}^{\mathrm{int},+}}[\llbracket \overrightarrow{\boldsymbol{\varphi}} \rrbracket+\overrightarrow{\boldsymbol{F}} \cdot \llbracket \boldsymbol{X} \rrbracket] \otimes \boldsymbol{M} \mathrm{d} A\right] \\
& =\frac{1}{V_{0}} \overrightarrow{\boldsymbol{F}} \cdot \int_{\partial \mathcal{B}_{0}^{\mathrm{pln},+}} \llbracket \boldsymbol{X} \rrbracket \otimes \boldsymbol{N}^{+} \mathrm{d} A+\frac{1}{h_{0}} \llbracket \overline{\boldsymbol{\varphi}} \rrbracket \otimes \boldsymbol{M}+\boldsymbol{M} \otimes \boldsymbol{M} \\
& =\underbrace{\overbrace{\partial \mathcal{B}_{0}^{\text {pln },+}}^{\widehat{\boldsymbol{F}} \cdot \sum \boldsymbol{N}^{+} \otimes \boldsymbol{N}^{+}}+\boldsymbol{M} \otimes \boldsymbol{M}}_{\overrightarrow{\boldsymbol{F}}}+\frac{1}{h_{0}} \llbracket \overline{\boldsymbol{\varphi}} \rrbracket \otimes \boldsymbol{M} \\
& =\overrightarrow{\boldsymbol{F}}+\frac{1}{h_{0}} \llbracket \overline{\boldsymbol{\varphi}} \rrbracket \otimes \boldsymbol{M},
\end{aligned}
$$


where we have used the results

$$
\begin{array}{cc}
\llbracket \boldsymbol{X} \rrbracket=l_{0}^{I} \boldsymbol{N}^{+, I} & \text { on } \partial \mathcal{B}_{0}^{+, I}, \\
\overrightarrow{\boldsymbol{F}} \cdot \boldsymbol{M}=\boldsymbol{M}, & \\
\int_{\partial \mathcal{B}_{0}^{+, I}}[\overrightarrow{\boldsymbol{F}} \cdot \llbracket \boldsymbol{X} \rrbracket] \otimes \boldsymbol{N}^{+, I} \mathrm{~d} A=V_{0} \overrightarrow{\boldsymbol{F}} \cdot\left[\boldsymbol{N}^{+, I} \otimes \boldsymbol{N}^{+, I}\right] .
\end{array}
$$

The result (35) agrees with that used by Hirschberger et al. [15] for the simplified case where $\overrightarrow{\boldsymbol{F}}=\boldsymbol{I}$ and was motivated by problems in the field of localised plasticity. ${ }^{3}$ Thus, the first term on the right-hand side of Eq. (35) accounts for the additional macroscopic kinematics associated with the continuum interface theory of Gurtin and Murdoch [1]. The second term is identical to that used by Hirschberger et al. [15] and is the portion of the measure of the effective macroscopic deformation gradient $\overline{\mathcal{F}}$ associated with the opening of the interface.

Remark. Consider the case where the macroscopic and microscopic axes coincide. The extension of the superficial interface deformation gradient $\widehat{\boldsymbol{F}}$ to $\mathbb{R}^{3}$ can be interpreted by considering the following matrix representation:

$$
\underbrace{\left[\begin{array}{ccc}
\widehat{F}_{11} & \widehat{F}_{12} & 0 \\
\widehat{F}_{21} & \widehat{F}_{22} & 0 \\
\widehat{F}_{31} & \widehat{F}_{32} & 1
\end{array}\right]}_{\overrightarrow{\boldsymbol{F}}} \cdot \underbrace{\left[\begin{array}{lll}
1 & 0 & 0 \\
0 & 1 & 0 \\
0 & 0 & 0
\end{array}\right]}_{\sum_{\partial \mathcal{B}_{0}^{\text {pln },+}} \boldsymbol{N}^{+} \otimes \boldsymbol{N}^{+}}+\underbrace{\left[\begin{array}{lll}
0 & 0 & 0 \\
0 & 0 & 0 \\
0 & 0 & 1
\end{array}\right]}_{\boldsymbol{M} \otimes \boldsymbol{M}}=\underbrace{\left[\begin{array}{lll}
\widehat{F}_{11} & \widehat{F}_{12} & 0 \\
\widehat{F}_{21} & \widehat{F}_{22} & 0 \\
\widehat{F}_{31} & \widehat{F}_{32} & 1
\end{array}\right]}_{\overrightarrow{\boldsymbol{F}}} .
$$

Note that $\sum_{\partial \mathcal{B}_{0}^{\text {pln },+}} \boldsymbol{N}^{+} \otimes \boldsymbol{N}^{+}=\overline{\mathbb{P}}_{0}(\widehat{\boldsymbol{X}})$.

The tensor $\widehat{\boldsymbol{F}}$ is a superficial, second-order tensor (see Section 2.2). The tensor $\overrightarrow{\boldsymbol{F}}$ is the extension of the superficial tensor $\widehat{\boldsymbol{F}}$ to the three-dimensional space occupied by the RVE. The variation of $\overrightarrow{\boldsymbol{F}}$, however, is a superficial tensor. Thus,

$$
\widehat{\boldsymbol{P}}: \delta \widehat{\boldsymbol{F}}=\widehat{\boldsymbol{P}}: \delta \overrightarrow{\boldsymbol{F}}
$$

\footnotetext{
${ }^{3}$ It is tacitly assumed throughout that the generalised macroscopic deformation $\widehat{\boldsymbol{\Xi}}$, when used at the microscopic scale, is expressed in terms of the coordinate system attached to the interface which is aligned with the microscopic coordinate system (see Hirschberger et al. [15] for a related discussion).
} 
In the absence of interface stretch $\overrightarrow{\boldsymbol{F}} \equiv \boldsymbol{I}$ and the boundary condition on $\partial \mathcal{B}_{0}^{\text {int, }} \pm$ given in Eq. (31) becomes

$$
\varphi:=\boldsymbol{X} \pm \frac{1}{2} \llbracket \bar{\varphi} \rrbracket .
$$

3.4.2. Hill-type averaging condition in the absence of interface stretch [15]

The Hill-type averaging condition and the resulting averaging relations in the absence of interface stretch (i.e. $\overrightarrow{\boldsymbol{F}} \equiv \boldsymbol{I}$ ) are now derived. This was the case considered by Hirschberger et al. [15].

Consider an RVE corresponding to a macroscopic point $\widehat{\boldsymbol{X}}$ on the interface $\overline{\mathcal{I}}_{0}$. The assumption of in-plane periodicity, made previously to obtain the relation between the effective macroscopic deformation gradient $\overline{\mathcal{F}}$ and the macroscopic kinematic quantities $\overrightarrow{\boldsymbol{F}}$ and $\llbracket \overline{\boldsymbol{\varphi}} \rrbracket$ in Eq. (35), will be shown to be in accordance with the Hill-type averaging condition.

From Eq. (35) and the ansatz on the microscopic motion (23) it follows that

$$
\begin{aligned}
\delta \boldsymbol{\varphi} & \equiv \underbrace{\left[\frac{1}{h_{0}} \llbracket \delta \overline{\boldsymbol{\varphi}} \rrbracket \boldsymbol{M}\right]}_{\delta \overline{\mathcal{F}}} \cdot \boldsymbol{X}+\delta \boldsymbol{w} \quad\left(\boldsymbol{w}=\mathbf{0} \text { on } \partial \mathcal{B}_{0}^{\mathrm{int}, \pm}\right), \\
\delta \bar{W}_{0}(\overline{\boldsymbol{X}}) & \equiv \widetilde{\boldsymbol{t}}_{0} \cdot \llbracket \delta \overline{\boldsymbol{\varphi} \rrbracket} .
\end{aligned}
$$

The Hill-type averaging condition follows as:

$$
\begin{gathered}
0 \doteq H=\frac{h_{0}}{V_{0}} \int_{\partial \mathcal{B}_{0}} \boldsymbol{t}_{0} \cdot \delta \boldsymbol{\varphi} \mathrm{d} A-\widetilde{\boldsymbol{t}}_{0} \cdot \llbracket \delta \overline{\boldsymbol{\varphi}} \rrbracket \\
=\left[\frac{1}{V_{0}} \int_{\partial \mathcal{B}_{0}^{\text {int }}} \boldsymbol{t}_{0} \otimes \boldsymbol{X} \mathrm{d} A \cdot \boldsymbol{M}\right] \cdot \llbracket \delta \overline{\boldsymbol{\varphi}} \rrbracket+\frac{h_{0}}{V_{0}} \int_{\partial \mathcal{B}_{0}^{\text {pln }}} \boldsymbol{t}_{0} \cdot \delta \boldsymbol{w} \mathrm{d} A-\widetilde{\boldsymbol{t}}_{0} \cdot \llbracket \delta \overline{\boldsymbol{\varphi}} \rrbracket \\
\Longrightarrow \quad \widetilde{\boldsymbol{t}}_{0}=\frac{1}{V_{0}} \int_{\partial \mathcal{B}_{0}^{\text {int }}} \boldsymbol{t}_{0} \otimes \boldsymbol{X} \mathrm{d} A \cdot \boldsymbol{M} \\
\Longrightarrow \quad 0=\int_{\partial \mathcal{B}_{0}^{\text {pln }}} \boldsymbol{t}_{0} \cdot \delta \boldsymbol{w} \mathrm{d} A
\end{gathered}
$$

The Hill-type averaging condition is thus satisfied if we choose periodic fluctuations and anti-periodic tractions on $\partial \mathcal{B}_{0}^{\text {pln }}$. Furthermore the macroscopic 
interface traction $\widetilde{\boldsymbol{t}}_{0}$ can be expressed as:

$$
\begin{aligned}
\widetilde{\boldsymbol{t}}_{0} & =\frac{1}{V_{0}} \int_{\partial \mathcal{B}_{0}^{\mathrm{int},+}} \boldsymbol{t}_{0}^{+} \otimes \llbracket \boldsymbol{X} \rrbracket \mathrm{d} A \cdot \boldsymbol{M} \\
& =\frac{1}{V_{0}}\left[\boldsymbol{f}_{0}^{3} \otimes \boldsymbol{l}_{0}^{3}\right] \cdot \boldsymbol{M} \\
& =\frac{h_{0}}{V_{0}} \boldsymbol{f}_{0}^{3} .
\end{aligned}
$$

Thus, only the stress resultant $\boldsymbol{f}_{0}^{3}$ (defined in Eq. (30)) acting on the surface $\partial \mathcal{B}_{0}^{\text {int, }}+$ need be determined in order to calculate the macroscopic interface traction $\widetilde{\boldsymbol{t}}_{0}$.

\subsubsection{Hill-type averaging condition accounting for interface stretch}

The Hill-type averaging condition and the resulting averaging relations in the presence of interface stretch are now derived.

From Eq. (35) and the ansatz on the microscopic motion (23) it follows that:

$$
\begin{aligned}
\delta \boldsymbol{\varphi} & \equiv \underbrace{\left[\delta \overrightarrow{\boldsymbol{F}}+\frac{1}{h_{0}} \llbracket \delta \overline{\boldsymbol{\varphi}} \rrbracket \otimes \boldsymbol{M}\right]}_{\delta \overline{\mathcal{F}}} \cdot \boldsymbol{X}+\delta \boldsymbol{w} \quad\left(\boldsymbol{w}=\mathbf{0} \text { on } \partial \mathcal{B}_{0}^{\text {int, }}\right), \\
\delta \bar{W}_{0}(\overline{\boldsymbol{X}}) & \equiv \widetilde{\boldsymbol{t}}_{0} \cdot \llbracket \delta \overline{\boldsymbol{\varphi}} \rrbracket+\widehat{\boldsymbol{P}}: \delta \widehat{\boldsymbol{F}} .
\end{aligned}
$$

The Hill-type averaging condition follows as:

$$
\begin{aligned}
& 0 \doteq H=\frac{h_{0}}{V_{0}} \int_{\partial \mathcal{B}_{0}} \boldsymbol{t}_{0} \cdot \delta \boldsymbol{\varphi} \mathrm{d} A-\widetilde{\boldsymbol{t}}_{0} \cdot \llbracket \delta \overline{\boldsymbol{\varphi}} \rrbracket-\widehat{\boldsymbol{P}}: \delta \widehat{\boldsymbol{F}} \\
& =\left[\frac{h_{0}}{V_{0}} \int_{\partial \mathcal{B}_{0}^{\text {pln }}} \boldsymbol{t}_{0} \otimes \boldsymbol{X} \mathrm{d} A\right]: \delta \widehat{\boldsymbol{F}}+\left[\frac{1}{V_{0}} \int_{\partial \mathcal{B}_{0}^{\text {int }}} \boldsymbol{t}_{0} \otimes \boldsymbol{X} \mathrm{d} A \cdot \boldsymbol{M}\right] \cdot \llbracket \delta \overline{\boldsymbol{\varphi}} \rrbracket \\
& +\frac{h_{0}}{V_{0}} \int_{\partial \mathcal{B}_{0}^{\mathrm{pln}}} \boldsymbol{t}_{0} \cdot \delta \boldsymbol{w} \mathrm{d} A-\widetilde{\boldsymbol{t}}_{0} \cdot \llbracket \delta \overline{\boldsymbol{\varphi}} \rrbracket-\widehat{\boldsymbol{P}}: \delta \widehat{\boldsymbol{F}} \\
& \Longrightarrow \quad \widetilde{\boldsymbol{t}}_{0}=\frac{1}{V_{0}} \int_{\partial \mathcal{B}_{0}^{\text {int }}} \boldsymbol{t}_{0} \otimes \boldsymbol{X} \mathrm{d} A \cdot \boldsymbol{M}, \\
& \Longrightarrow \quad \widehat{\boldsymbol{P}}=\frac{h_{0}}{V_{0}} \int_{\partial \mathcal{B}_{0}^{\mathrm{pln}}} \boldsymbol{t}_{0} \otimes \boldsymbol{X} \mathrm{d} A, \\
& \Longrightarrow \quad 0=\int_{\partial \mathcal{B}_{0}^{\text {pln }}} \boldsymbol{t}_{0} \cdot \delta \boldsymbol{w} \mathrm{d} A \text {. }
\end{aligned}
$$


Once again, the Hill-type averaging condition is satisfied if we choose periodic fluctuations and anti-periodic tractions on $\partial \mathcal{B}_{0}^{\text {pln }}$, as assumed previously. Furthermore, the macroscopic interface traction $\widetilde{\boldsymbol{t}}_{0}$ and the interface stress $\widehat{\boldsymbol{P}}$ are conveniently calculated as follows:

$$
\begin{aligned}
\widetilde{\boldsymbol{t}}_{0} & =\frac{1}{V_{0}} \int_{\partial \mathcal{B}_{0}^{\mathrm{int},+}} \boldsymbol{t}_{0}^{+} \otimes \llbracket \boldsymbol{X} \rrbracket \mathrm{d} A \cdot \boldsymbol{M} \\
= & \frac{h_{0}}{V_{0}} \boldsymbol{f}_{0}^{3}, \\
\widehat{\boldsymbol{P}}_{0} & =\frac{1}{V_{0}} \int_{\partial \mathcal{B}_{0}^{\mathrm{pln},+}} \boldsymbol{t}_{0}^{+} \otimes \llbracket \boldsymbol{X} \rrbracket \mathrm{d} A \\
& =\frac{1}{V_{0}} \sum_{\alpha=1}^{2}\left[\boldsymbol{f}_{0}^{\alpha} \otimes \boldsymbol{l}_{0}^{\alpha}\right] .
\end{aligned}
$$

\subsection{Discussion}

Comparing the relations for the macroscopic Piola stress in the bulk $\overline{\boldsymbol{P}}$ and on the interface $\widehat{\boldsymbol{P}}$, given in Eq. (25) and (38) respectively, it is obvious that they have the same structure. The macroscopic interface Piola stress, however, is obtained by considering only the in-plane faces of the RVE. The expressions for the macroscopic interface traction $\widetilde{\boldsymbol{t}}_{0}$ for an interface with and without stretch, given in Eq. (36) and (37), are identical.

\section{Computational homogenisation}

The focus of this contribution is on determining the response of the microstructure assuming the macroscopic kinematic quantities $\widehat{\boldsymbol{\Xi}}$ to be known. The numerical solution of the fully-coupled micro-macro problem will be addressed in a later contribution, but it will obviously have some similarities to the scheme developed by Hirschberger et al. [15]. The objective of the simulation at the microscopic scale is thus to determine the averaged macroscopic kinetic quantities $\widehat{\Sigma}$ and the macroscopic tangent $\widehat{\mathcal{A}}$ for given macroscopic kinematic quantities $\widehat{\boldsymbol{\Xi}}$.

Consider a partition of the time domain $\mathbb{T}$ into $n_{\mathrm{ts}}$ intervals

$$
\mathbb{T}=\bigcup_{\mathrm{n}=0}^{n_{\mathrm{ts}}}\left[t_{\mathrm{n}}, t_{\mathrm{n}+1}\right],
$$

whereby $\Delta t:=t_{\mathrm{n}+1}-t_{\mathrm{n}}$ denotes the duration of the time increment. The complete state of the system at time $t_{n}$ is assumed to be known. An iterative 
scheme is then used to solve the fully-discrete system of equations at time $t_{\mathrm{n}+1}$. The current iteration is denoted (i) and the increment of a quantity between iterations is defined by

$$
\mathrm{d}\{\bullet\}:=\{\bullet\}_{\mathrm{n}+1}^{(\mathrm{i}+1)}-\{\bullet\}_{\mathrm{n}+1}^{(\mathrm{i})}=\{\bullet\}^{(\mathrm{i}+1)}-\{\bullet\}^{(\mathrm{i})} .
$$

The nonlinear microscopic boundary value problem (V), given in Eq. (18), is approximated here using the finite element method. The material configuration $\mathcal{B}_{0}$ is subdivided into $n_{\mathrm{e}}$ non-overlapping hexahedral domains as $\mathcal{B}_{0} \approx \mathcal{B}_{0}^{h}=\bigcup_{\mathrm{e}=1}^{n_{\mathrm{e}}} \mathcal{B}_{0}^{\mathrm{e}}$, where $\mathcal{B}_{0}^{\mathrm{e}}$ is an individual subdomain (finite element). The interpolation of material particles $\boldsymbol{X}$ within the reference domain and the approximation of the motion $\boldsymbol{\varphi}$ follow as per conventional Galerkin finite element procedure based upon the isoparametric concept; that is,

$$
\boldsymbol{X} \approx \boldsymbol{X}^{h}(\boldsymbol{\xi})=\sum_{\mathrm{I}=1}^{n_{\mathrm{n}}} N_{\mathrm{I}}(\boldsymbol{\xi}) \boldsymbol{X}_{\mathrm{I}} \quad \text { and } \quad \boldsymbol{\varphi} \approx \boldsymbol{\varphi}^{h}(\boldsymbol{\xi})=\sum_{\mathrm{I}=1}^{n_{\mathrm{n}}} N_{\mathrm{I}}(\boldsymbol{\xi}) \boldsymbol{\varphi}_{\mathrm{I}}
$$

where $N_{\mathrm{I}}$ is the shape function, $\boldsymbol{X}_{\mathrm{I}}$ the material coordinates, and $\boldsymbol{\varphi}_{\mathrm{I}}$ the motion, all associated with node I. The variable $n_{\mathrm{n}}$ denotes the number of nodes. Coordinates within the isoparametric space are denoted $\boldsymbol{\xi} \in[-1,1]^{3}$. Identical interpolations are used for the test functions and trial solutions in accordance with the classical Bubnov-Galerkin approach.

The fully-discrete variational counterpart to the nonlinear microscopic boundary value problem $(\mathrm{V})$ at time $t_{\mathrm{n}+1}$ is thus given by:

$$
\boldsymbol{r}_{\mathrm{I}}\left(\boldsymbol{\varphi}^{h}\right):=\int_{\mathcal{B}_{0}^{h}} \boldsymbol{P}_{\mathrm{n}+1}^{h}\left(\boldsymbol{\varphi}^{h}\right) \cdot \operatorname{Grad} N_{\mathrm{I}} \mathrm{d} V=\mathbf{0} \quad \text { for } \mathrm{I}=1,2, \ldots, n_{\mathrm{n}},
$$

where $\boldsymbol{r}_{\mathrm{I}} \in \mathbb{R}^{3}$ is the residual vector associated with node I. The global residual vector $\mathbf{r}$ is obtained from the nodal contributions $\boldsymbol{r}_{\mathrm{I}}$ following standard procedure.

A Newton-Raphson scheme is used to solve the resulting system of equations subject to the boundary conditions specified in Eq. (31)-(34) for the global vector of nodal motions $\boldsymbol{\varphi}$; that is

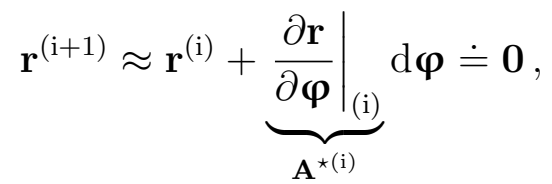


where $\mathbf{A}^{\star}$ is the total (algorithmic) microscopic tangent matrix with nodal contributions given by

$$
\mathbf{A}_{\mathrm{IJ}}^{\star(\mathrm{i})}:=\int_{\mathcal{B}_{0}^{h}} \operatorname{Grad} N_{\mathrm{I}} \cdot[\underbrace{\left.\frac{\partial \boldsymbol{P}^{h}}{\partial \boldsymbol{F}^{h}}\right|_{(\mathrm{i})}}_{\mathcal{A}^{(\mathrm{i})}} \cdot \operatorname{Grad} N_{\mathrm{J}}] \mathrm{d} V,
$$

and $\mathcal{A}$ is the (microscopic) tangent constitutive tensor. The boundary conditions are imposed on Eq. (41) via linear constraints [49]. Alternative approaches include the penalty method [14] and the method of Lagrange multipliers $[13,48]$. The triangulation of the domain is, for the sake of simplicity, periodic on the boundaries; non-periodic triangulations have been considered in [14]. It should be noted that no degrees of freedom need to be fictitiously constrained to prevent rigid-body translations as is done for the conventional homogenisation of a point within the macroscopic bulk; the Dirichlet conditions on $\partial \mathcal{B}_{0}^{\text {int }}$ given in Eq. (31) suffice.

Conceptually it proves convenient to consider the full microscopic tangent matrix, denoted $\mathbf{A}$, corresponding to $\mathbf{A}^{\star}$ at the final (i.e. converged) Newton-Raphson iteration. The tangent matrix $\mathbf{A}$ is partitioned such that (independent) nodes on the positive boundaries (denoted by the set $\eta^{+}=\left\{\mathrm{I}: \boldsymbol{X}_{\mathrm{I}} \in \partial \mathcal{B}_{0}^{+}\right\}$where $\left.\mathrm{I}=1,2, \ldots, n_{\mathrm{n}}\right)$ appear before the remaining (dependent) nodes on the negative boundaries and in the interior in the global nodal ordering. That is,

$$
\mathbf{A}:=\left[\begin{array}{cc}
\mathbf{A}_{a a} & \mathbf{A}_{a b} \\
\mathbf{A}_{b a} & \mathbf{A}_{b b}
\end{array}\right],
$$

where $\mathbf{A}_{a a}$ and $\mathbf{A}_{b b}$ are associated with the independent and dependent nodes, respectively. Following [14], a perturbation in the motion of the independent nodes $\delta \boldsymbol{\varphi}_{a}$ is related to a perturbation in the corresponding reaction forces $\delta \mathbf{f}_{a}$ as

$$
\underbrace{\left[\mathbf{A}_{a a}-\mathbf{A}_{a b} \cdot\left[\mathbf{A}_{b b}\right]^{-1} \cdot \mathbf{A}_{b a}\right]}_{\mathbf{A}^{+}} \cdot \delta \boldsymbol{\varphi}_{a}=\delta \mathbf{f}_{a},
$$

where $\mathbf{A}^{+}$is the Schur complement of the matrix $\mathbf{A}$.

Furthermore, it proves convenient to consider the set $\eta^{+}$to be the union of the two sets $\eta^{\text {pln,+ }}$ and $\eta^{\text {int,+ }}$ corresponding to nodes on the surfaces $\partial \mathcal{B}_{0}^{\text {pln,+}}$ and $\partial \mathcal{B}_{0}^{\text {int, }+}$, respectively. The set of in-plane nodes on the positive surfaces are further categorised depending on the direction of the outward 
surface normal relative to the basis vectors $\boldsymbol{E}_{\alpha}$ as $\eta_{1}^{\text {pln, }+}$ and $\eta_{2}^{\text {pln,+ }}$; i.e. $\eta_{\alpha}^{\text {pln },+}:=\left\{\mathrm{I}: \boldsymbol{X}_{I} \in \partial \mathcal{B}_{0}^{\text {pln, },}: \boldsymbol{N}^{+}\left(\boldsymbol{X}_{\mathrm{I}}\right) \cdot \boldsymbol{E}_{\alpha}=1\right\}$ for $\alpha=1,2$. The condensed expression given in Eq. (42) can therefore be further specialised to nodes in $\eta^{\text {int, }+}$ and $\eta_{\alpha}^{\text {pln,+}}$, respectively, as

$$
\begin{aligned}
\mathbf{A}_{\text {int }}^{+} \cdot \delta \boldsymbol{\varphi}_{\text {int }} & =\delta \mathbf{f}_{\text {int }}, \\
\mathbf{A}_{\mathrm{pln}}^{+, \alpha} \cdot \delta \boldsymbol{\varphi}_{\mathrm{pln}}^{\alpha} & =\delta \mathbf{f}_{\mathrm{pln}}^{\alpha} \quad \text { for } \alpha=1,2 .
\end{aligned}
$$

\subsection{Computation of the macroscopic kinetic quantities}

The reaction forces for all nodes in $\eta^{+}$is known from the solution of the finite element problem. The approximations of the macroscopic interface traction $\widetilde{\boldsymbol{t}}_{0}^{h}$ and the interface stress $\widehat{\boldsymbol{P}}^{h}$ given in Eq. (39)-(40) are thus computed as:

$$
\begin{aligned}
\widetilde{\boldsymbol{t}}_{0}^{h} & =\frac{h_{0}}{V_{0}} \sum_{\mathrm{I} \in \eta^{\mathrm{int},+}}\left[\boldsymbol{f}_{\mathrm{int}}\right]_{\mathrm{I}}, \\
\widehat{\boldsymbol{P}}^{h} & =\frac{1}{V_{0}} \sum_{\alpha=1}^{2} \sum_{\mathrm{I} \in \eta_{\alpha}^{\mathrm{pln},+}}\left[\left[\boldsymbol{f}_{\mathrm{pln}}^{\alpha}\right]_{\mathrm{I}} \otimes \boldsymbol{l}_{0}^{\alpha}\right] .
\end{aligned}
$$

\subsection{Computation of the macroscopic tangent}

The numerical approximation to the macroscopic tangent $\widehat{\mathcal{A}}$, defined in Eq. (14), is computed from an incremental expression of the macroscopic interface traction $\widetilde{\boldsymbol{t}}_{0}^{h}$ and the interface stress $\widehat{\boldsymbol{P}}^{h}$ given in Eq. (43)-(44) and 
the boundary conditions given in Eq. (31)-(34) as follows:

$$
\begin{gathered}
\delta \widetilde{\boldsymbol{t}}_{0}^{h}=\frac{h_{0}}{V_{0}} \sum_{\mathrm{I} \in \eta^{\text {int },+}}\left[\delta \boldsymbol{f}_{\mathrm{int}}\right]_{\mathrm{I}} \\
=\frac{h_{0}}{V_{0}} \sum_{\mathrm{I} \in \eta^{\mathrm{int},+}} \sum_{\mathrm{J} \in \eta^{\mathrm{int},+}}\left[\mathbf{A}_{\mathrm{int}}^{+}\right]_{\mathrm{IJ}} \cdot\left[\delta \boldsymbol{\varphi}_{\mathrm{int}}\right]_{\mathrm{J}} \\
=\frac{h_{0}}{V_{0}} \sum_{\mathrm{I} \in \eta^{\mathrm{int},+}} \sum_{\mathrm{J} \in \eta^{\mathrm{int},+}}\left[\mathbf{A}_{\mathrm{int}}^{+}\right]_{\mathrm{IJ}} \cdot\left[\left[\frac{1}{2} \llbracket \delta \overline{\boldsymbol{\varphi}} \rrbracket+\delta \overrightarrow{\boldsymbol{F}} \cdot \boldsymbol{X}_{\mathrm{J}}\right]\right], \\
\delta \widehat{\boldsymbol{P}}^{h}=\frac{1}{V_{0}} \sum_{\mathrm{I} \in \eta^{\mathrm{pln},+}}\left[\left[\delta \boldsymbol{f}_{\mathrm{pln}}\right]_{\mathrm{I}} \otimes \boldsymbol{l}_{0}^{\alpha}\right] \\
=\frac{1}{V_{0}} \sum_{\alpha=1}^{2} \sum_{\mathrm{I} \in \eta_{\alpha}^{\mathrm{pln},+}} \sum_{\mathrm{J} \in \eta_{\alpha}^{\mathrm{pln},+}}\left[\left[\mathbf{A}_{\mathrm{pln}}^{+, \alpha}\right]_{\mathrm{IJ}} \cdot\left[\delta \boldsymbol{\varphi}_{\mathrm{pln}}^{\alpha}\right]_{\mathrm{J}}\right] \otimes \boldsymbol{l}_{0}^{\alpha} \\
=\frac{1}{V_{0}} \sum_{\alpha=1}^{2} \sum_{\mathrm{I} \in \eta_{\alpha}^{\mathrm{pln},+}} \sum_{\mathrm{J} \in \eta_{\alpha}^{\mathrm{pln},+}}\left[\left[\mathbf{A}_{\mathrm{pln}}^{+, \alpha}\right]_{\mathrm{IJ}} \cdot\left[\frac{\left[X_{3}\right]_{\mathrm{J}}}{h_{0}} \llbracket \delta \overline{\boldsymbol{\varphi}} \rrbracket+\delta \overrightarrow{\boldsymbol{F}} \cdot \boldsymbol{X}_{\mathrm{J}}\right]\right] \otimes \boldsymbol{l}_{0}^{\alpha} .
\end{gathered}
$$

Consequently one obtains the approximation to the macroscopic tangent, denoted $\widehat{\mathcal{A}}^{h}$, from Eq. (14) and the preceding relations as

$$
\begin{aligned}
\delta \widehat{\boldsymbol{\Sigma}}^{h} & =\widehat{\mathcal{A}}^{h}: \delta \widehat{\boldsymbol{\Xi}}^{h} \\
& =\left[\begin{array}{cc}
\partial_{\llbracket \delta \overline{\boldsymbol{\varphi}}} \delta \widetilde{\boldsymbol{t}}_{0}^{h} & \partial_{\delta \overrightarrow{\boldsymbol{F}}} \delta \widetilde{\boldsymbol{t}}_{0}^{h} \\
\partial_{\llbracket \delta \overline{\boldsymbol{\varphi}} \rrbracket} \delta \widehat{\boldsymbol{P}}^{h} & \partial_{\delta \overrightarrow{\boldsymbol{F}}} \delta \widehat{\boldsymbol{P}}^{h}
\end{array}\right]: \delta \widehat{\boldsymbol{\Xi}}^{h},
\end{aligned}
$$

where

$$
\begin{aligned}
\partial_{\llbracket \delta \overline{\boldsymbol{\varphi}} \rrbracket} \delta \widetilde{\boldsymbol{t}}_{0}^{h} & =\frac{h_{0}}{2 V_{0}} \sum_{\mathrm{I} \in \eta^{\mathrm{int},+}} \sum_{\mathrm{J} \in \eta^{\mathrm{int},+}}\left[\mathbf{A}_{\mathrm{int}}^{+}\right]_{\mathrm{IJ}}, \\
\partial_{\delta \overrightarrow{\boldsymbol{F}}} \delta \widetilde{\boldsymbol{t}}_{0}^{h} & =\frac{h_{0}}{V_{0}} \sum_{\mathrm{I} \in \eta^{\mathrm{int},+}} \sum_{\mathrm{J} \in \eta^{\mathrm{int},+}}\left[\mathbf{A}_{\mathrm{int}}^{+}\right]_{\mathrm{IJ}} \otimes \boldsymbol{X}_{\mathrm{J}}, \\
\partial_{\llbracket \delta \overline{\boldsymbol{\varphi}} \rrbracket} \delta \widehat{\boldsymbol{P}}^{h} & =\frac{1}{V_{0}} \sum_{\alpha=1}^{2} \sum_{\mathrm{I} \in \eta_{\alpha}^{\mathrm{pln},+}} \sum_{\mathrm{J} \in \eta_{\alpha}^{\mathrm{pln},+}} \frac{\left[X_{3}\right]_{\mathrm{J}}}{h_{0}}\left[\mathbf{A}_{\mathrm{pln}}^{+, \alpha}\right]_{\mathrm{IJ}} \bar{\otimes} \boldsymbol{l}_{0}^{\alpha}, \\
\partial_{\delta \overrightarrow{\boldsymbol{F}}} \delta \widehat{\boldsymbol{P}}^{h} & =\frac{1}{V_{0}} \sum_{\alpha=1}^{2} \sum_{\mathrm{I} \in \eta_{\alpha}^{\mathrm{pln},+}} \sum_{\mathrm{J} \in \eta_{\alpha}^{\mathrm{pln},+}}\left[\mathbf{A}_{\mathrm{pln}}^{+, \alpha}\right]_{\mathrm{IJ}} \bar{\otimes}\left[\boldsymbol{l}_{0}^{\alpha} \otimes \boldsymbol{X}_{\mathrm{J}}\right] .
\end{aligned}
$$




\section{Numerical results}

The objective of this section is to illustrate the previously derived theory using a series of representative numerical examples. The finite element implementation uses the open-source library deal. II [49]. Various permutations of the macroscopic kinematic parameters $\widehat{\boldsymbol{F}}$ and $\llbracket \overline{\boldsymbol{u}} \rrbracket$ are chosen and the respective conjugate kinetic quantities $\widehat{\boldsymbol{P}}$ and $\widetilde{\boldsymbol{t}}_{0}$ determined from the numerical solution of the problem (V) using the finite element method. ${ }^{4}$

The material configuration of the RVE is a unit cube containing a spherical void or stiff inclusion of diameter 0.5 as depicted in Fig. 5. The constitutive response of the microscopic problem is approximated using a neoHookean model. The Poisson's ratio $\nu$ and shear modulus $\mu$ of the material surrounding the void are $\nu=0.3$ and $\mu=80.194$, respectively. The shear modulus of the stiff inclusion is ten times that of the surrounding material.

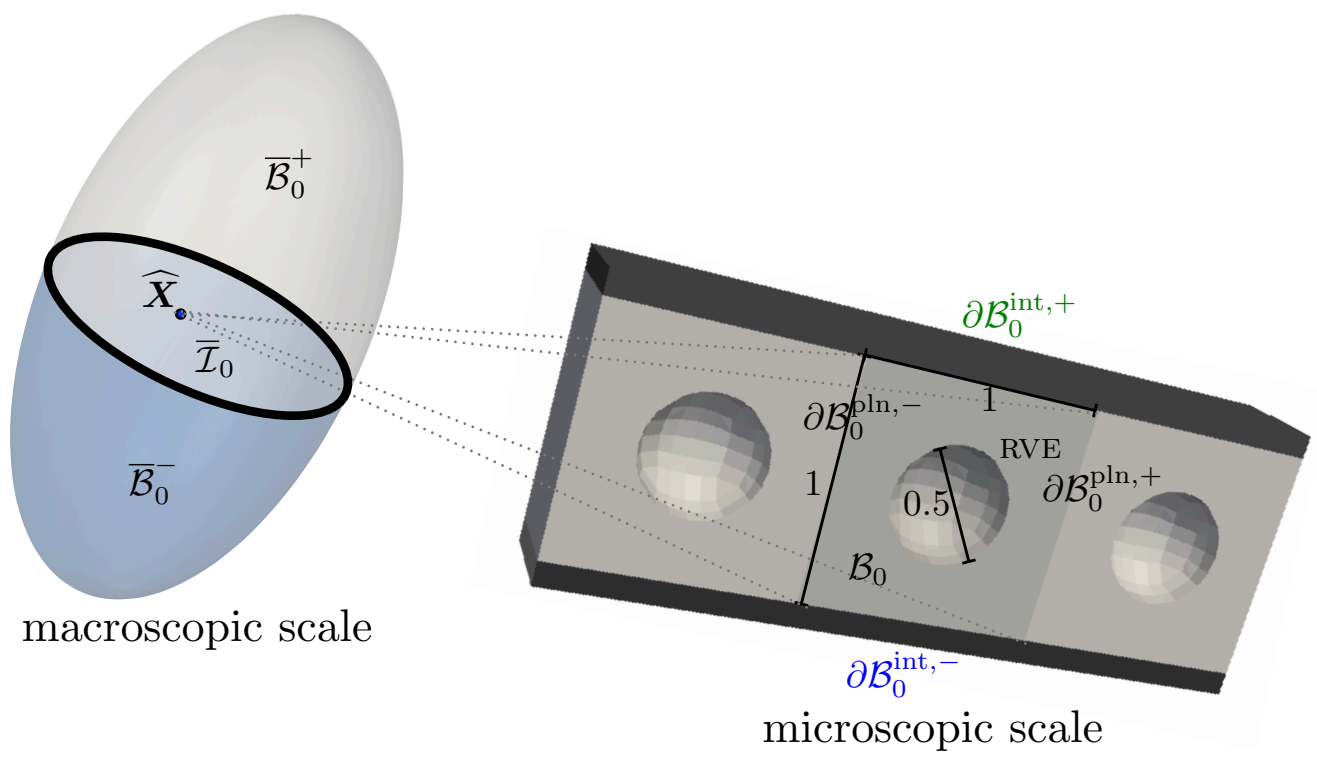

Figure 5: Cross section through the material configuration of the RVE containing a spherical void or a stiff inclusion.

\subsection{Microstructure with a void}

The deformed microscopic configurations corresponding to various macroscopic loading conditions are shown in Fig. 6 and the results summarised in

\footnotetext{
${ }^{4}$ The superscript $h$ denoting a finite-dimensional approximation is omitted here and henceforth for the sake of brevity.
} 
Table 1.

The results in Fig. 6(a)-(c) correspond to setting $\overrightarrow{\boldsymbol{F}} \equiv \boldsymbol{I}$. The upper and lower surfaces of the RVE, which are conceptually attached to the surrounding macroscopic bulk, are thus not allowed to stretch. In the loading case depicted in Fig. 6(a) the in-plane faces of the RVE are prevented from deforming in the plane due to the periodicity constraint. This, in turn, causes the spherical void to undergo significant deformation. For the loading case depicted in Fig. 6(b), the upper and lower surfaces of the RVE translate an equal distance in opposing horizontal directions. The loading scenario in Fig. 6(c) corresponds to combining the applied displacement jumps in (a) and (b). Critically, and unlike in the theory proposed by Hirschberger et al. [15], even in the absence of in-plane stretch one obtains a non-zero macroscopic interface stress $\widehat{\boldsymbol{P}}$ (see Table 1 ).

Now consider the case depicted in Fig. 6(d) where only an interface deformation gradient $\widehat{\boldsymbol{F}}$ is applied. The ability of the RVE to stretch is clear. As in loading scenario (a), the in-plane faces of the RVE remain vertical. In Fig. 6(e), both a displacement jump and a macroscopic interface deformation gradient are applied. The periodic motion of the in-plane faces is clear, as is the large amount of deformation of the void.

The RVE can also undergo a shear in the $X-Z$ plane by specifying $\widehat{\boldsymbol{F}}$ as in Fig. 6(f). This allows the mid-plane of the RVE to rotate relative to its position in the undeformed configuration. Crucially, however, the upper and lower surfaces remain parallel. Thus, due to the first-order ansatz (23), the RVE can not reproduce bending modes. Furthermore, the jump in the microscopic displacement field $\llbracket \boldsymbol{\varphi} \rrbracket$ between the surfaces $\partial \mathcal{B}_{0}^{\text {int, } \pm}$ and hence in the macroscopic displacement fields $\llbracket \bar{\varphi} \rrbracket$, is zero; the interface deformation gradient $\widehat{\boldsymbol{F}}$ and the macroscopic displacement jump $\llbracket \overline{\boldsymbol{\varphi}} \rrbracket$ are independent descriptors of the deformation of the interface.

\subsection{Microstructure with a spherical inclusion}

The void is now replaced with a stiff inclusion and the same series of numerical tests performed. The deformed configurations corresponding to the loading conditions are shown in Fig. 7 and the effective macroscopic kinetic quantities given in Table 1.

In contrast to the previous example involving a spherical void, the majority of the deformation now occurs within the material surrounding the stiff inclusion. This leads to, in general, higher values for the calculated macroscopic interface traction $\widetilde{\boldsymbol{t}}_{0}$ and interface stress $\widehat{\boldsymbol{P}}$ for the same applied macroscopic kinematic quantities. 
(a)

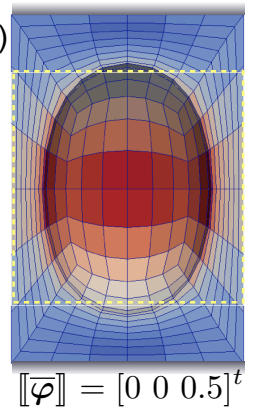

(d)

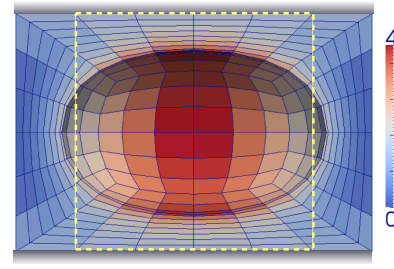

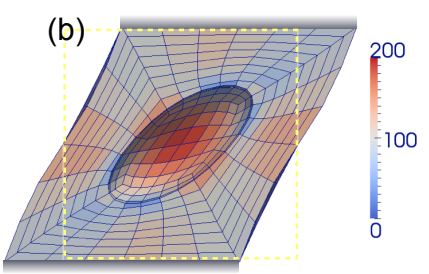

$\llbracket \bar{\varphi} \rrbracket=\left[\begin{array}{lll}0.5 & 0 & 0\end{array}\right]^{t}$
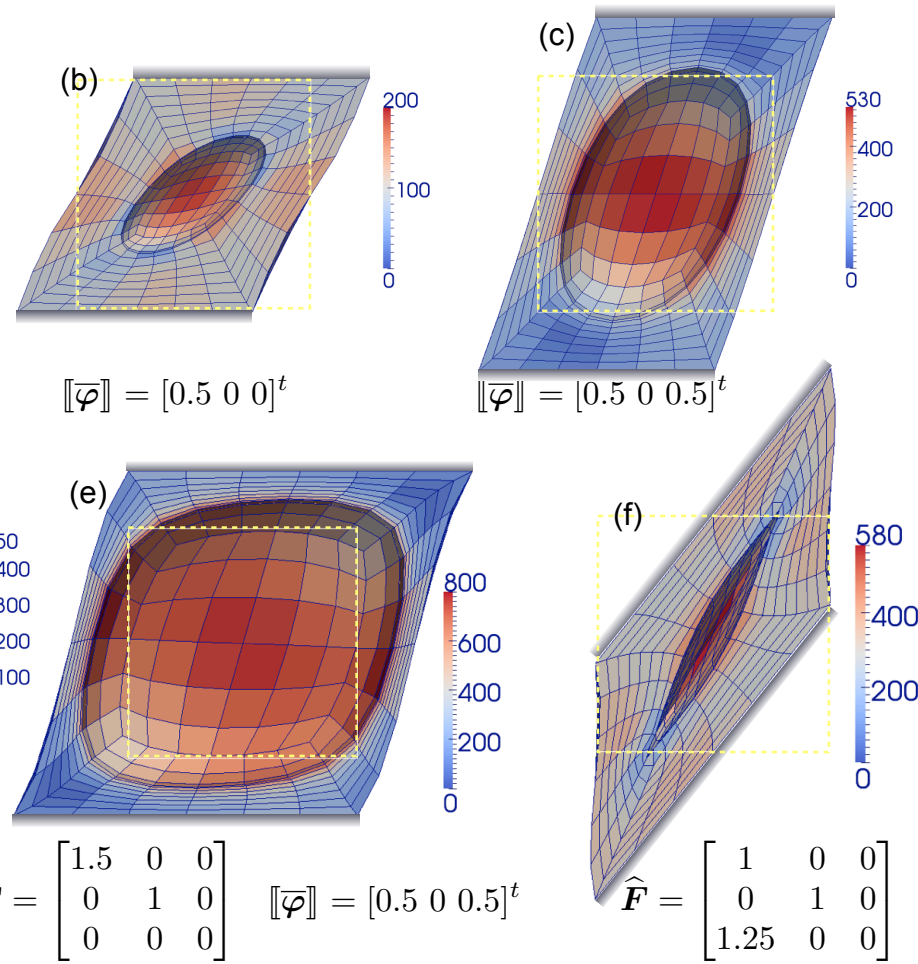

Figure 6: Cross section through the three-dimensional deformed RVE containing a void for various macroscopic loading conditions. The results are coloured by the Frobenius norm of the Kirchhoff stress $\boldsymbol{\tau}:=\boldsymbol{P} \cdot \boldsymbol{f}^{t}$. The material configuration is indicated by a dashed (yellow) line and all results have the same geometric scale.

The macroscopic kinematic data and the subsequent microscopic response shown in Fig. 8 illustrate the three-dimensional nature of the formulation.

\subsection{Discussion}

The coupling between the prescribed kinematic data $\widehat{\boldsymbol{\Xi}}$ and the resulting kinetic response $\widehat{\boldsymbol{\Sigma}}$ is evident from the results given in Table 1. That this should be the case is clear from the fully-coupled macroscopic tangent $\mathcal{A}^{h}$ derived in Section 4.2 . 
Table 1: Summary of the calculated averaged kinetic response arising from the prescribed kinematic data for a RVE with a spherical void, and with a stiff inclusion.

\begin{tabular}{|c|c|c|c|c|c|c|c|c|c|c|}
\hline \multirow[b]{2}{*}{$\begin{array}{l}\text { Load } \\
\text { case }\end{array}$} & \multirow[b]{2}{*}{$\llbracket \bar{\varphi} \rrbracket$} & & & \multicolumn{3}{|c|}{ Void } & \multicolumn{4}{|c|}{ Stiff inclusion } \\
\hline & & \multicolumn{2}{|c|}{$\widehat{\boldsymbol{F}}$} & $\widetilde{\boldsymbol{t}}_{0}$ & \multicolumn{2}{|r|}{$\widehat{P}$} & $\widetilde{\boldsymbol{t}}_{0}$ & \multicolumn{3}{|c|}{$\widehat{P}$} \\
\hline \multirow{3}{*}{ (a) } & 0 & 1 & $\begin{array}{ll}0 & 0\end{array}$ & 0 & {$[76$} & $\left.\begin{array}{ll}0 & 0\end{array}\right]$ & 0 & {$[200$} & 0 & \\
\hline & 0 & & 10 & 0 & & $76 \quad 0$ & 0 & 0 & 200 & 0 \\
\hline & 0.5 & 0 & $\left.\begin{array}{ll}0 & 0\end{array}\right]$ & 100 & 0 & $\left.\begin{array}{ll}0 & 0\end{array}\right]$ & 192 & 0 & 0 & 0 \\
\hline \multirow{3}{*}{ (b) } & {$[0.5]$} & & $\begin{array}{ll}0 & 0\end{array}$ & {$[36]$} & {$[-3$} & $\left.\begin{array}{ll}0 & 0\end{array}\right]$ & {$[45]$} & {$[-9$} & 0 & \\
\hline & 0 & & $\begin{array}{ll}1 & 0\end{array}$ & 0 & & $\begin{array}{ll}-4 & 0\end{array}$ & 0 & 0 & -8 & 0 \\
\hline & 0 & 0 & $\left.\begin{array}{ll}0 & 0\end{array}\right]$ & $-4]$ & 39 & $\begin{array}{ll}0 & 0\end{array}$ & {$[-8]$} & 47 & 0 & $0]$ \\
\hline \multirow{3}{*}{ (c) } & {$[0.5]$} & & $\begin{array}{ll}0 & 0\end{array}$ & {$[31]$} & {$[74$} & $\begin{array}{ll}0 & 0\end{array}$ & $34]$ & $\lceil 194$ & 0 & $0]$ \\
\hline & 0 & & $\begin{array}{ll}1 & 0\end{array}$ & 0 & & $\begin{array}{ll}74 & 0\end{array}$ & 0 & 0 & 194 & 0 \\
\hline & 0.5 & 0 & $\left.\begin{array}{ll}0 & 0\end{array}\right]$ & 99 & $\lfloor-4$ & $\left.\begin{array}{ll}0 & 0\end{array}\right]$ & [188] & -43 & 0 & 0 \\
\hline \multirow{3}{*}{ (d) } & {$[0]$} & {$[1.5$} & $\begin{array}{ll}0 & 0\end{array}$ & $0]$ & {$[105$} & $\left.\begin{array}{ll}0 & 0\end{array}\right]$ & {$[0]$} & {$[193$} & 0 & $0]$ \\
\hline & 0 & & $\begin{array}{ll}1 & 0\end{array}$ & 0 & 0 & $\begin{array}{ll}77 & 0\end{array}$ & 0 & 0 & 202 & 0 \\
\hline & {$[0]$} & 0 & $\begin{array}{ll}0 & 0\end{array}$ & 74 & L 0 & $\left.\begin{array}{ll}0 & 0\end{array}\right]$ & 202 & 0 & 0 & $0]$ \\
\hline \multirow{3}{*}{ (e) } & {$[0.5]$} & {$[1.5$} & $\left.\begin{array}{ll}0 & 0\end{array}\right]$ & 29 & 127 & $\left.\begin{array}{ll}0 & 0\end{array}\right]$ & {$[26]$} & 507 & 0 & 0 \\
\hline & 0 & & 10 & 0 & 0 & 1110 & 0 & 0 & 696 & 0 \\
\hline & $0.5]$ & 0 & $\begin{array}{ll}0 & 0\end{array}$ & 119 & -17 & $\left.\begin{array}{ll}0 & 0\end{array}\right]$ & 508 & -144 & 0 & 0 \\
\hline \multirow{3}{*}{ (f) } & {$[0]$} & & $\left.\begin{array}{ll}0 & 0\end{array}\right]$ & $121]$ & {$[-37$} & 0 & {$[179]$} & {$[-35$} & 0 & 07 \\
\hline & 0 & & 10 & 0 & 0 & $-28 \quad 0$ & 0 & 0 & -45 & 0 \\
\hline & {$[0$} & 1.25 & $0 \quad 0]$ & $-27\rfloor$ & 97 & $\begin{array}{ll}0 & 0\end{array}$ & {$[-52]$} & 104 & 0 & 0 \\
\hline
\end{tabular}


(a)

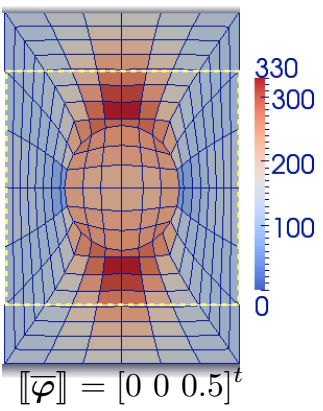

(d)

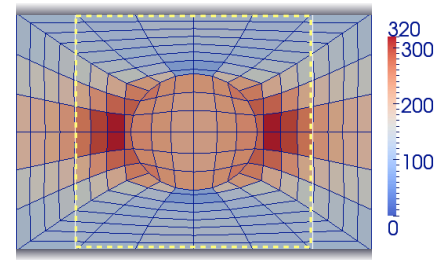

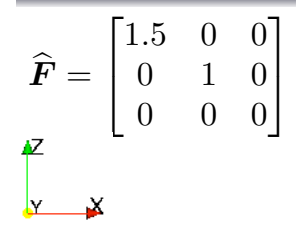

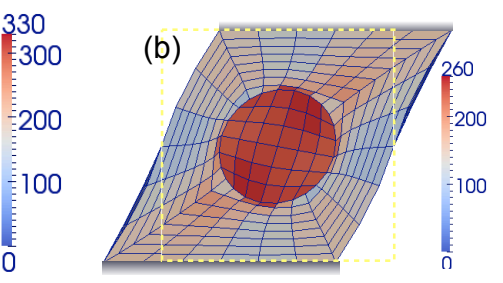

$\llbracket \bar{\varphi} \rrbracket=\left[\begin{array}{lll}0.5 & 0 & 0\end{array}\right]^{t}$ (c)

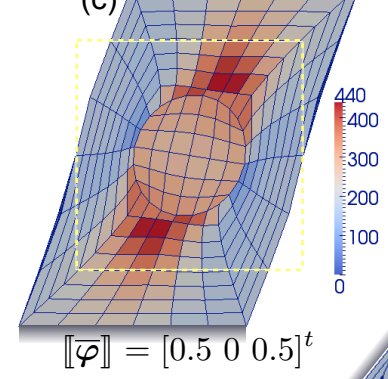

Figure 7: Cross section through the three-dimensional deformed RVE containing a stiff inclusion for various macroscopic loading conditions. The results are coloured by the Frobenius norm of the Kirchhoff stress. The material configuration is indicated by a dashed (yellow) line and all results have the same geometric scale. 


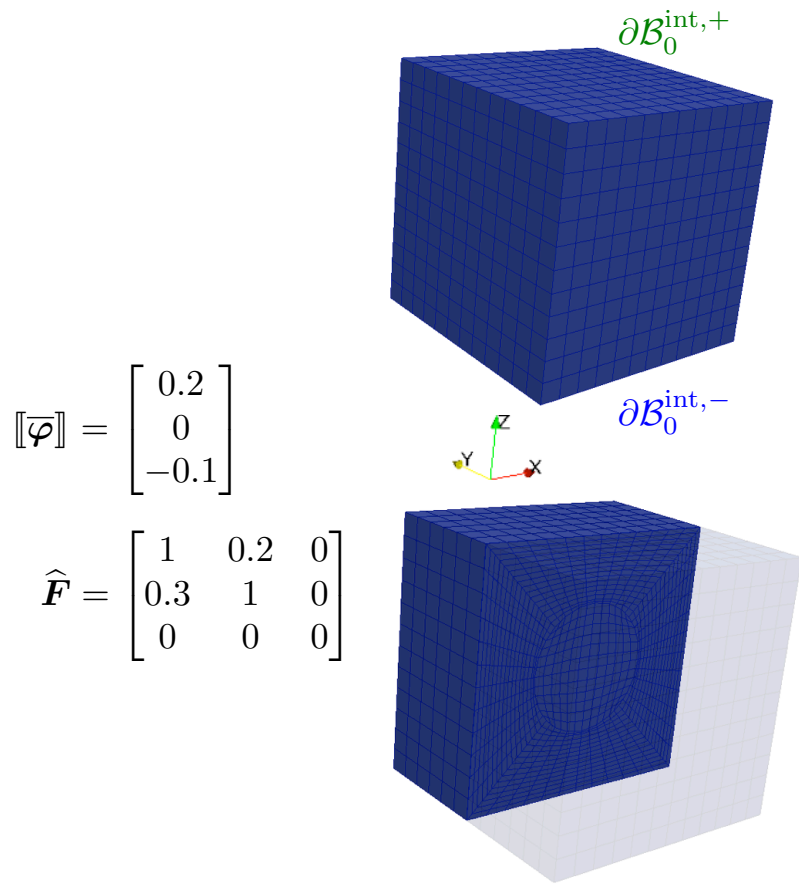

$\mathcal{B}_{0}$

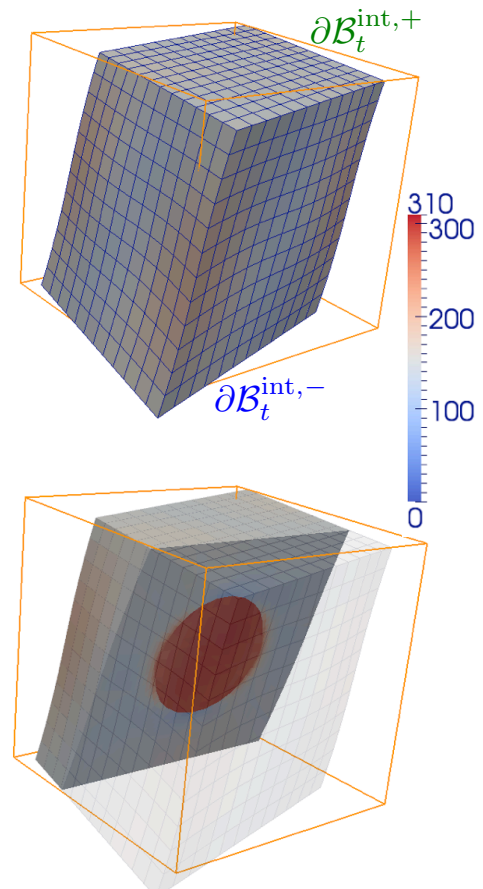

$\mathcal{B}_{t}$

Figure 8: The material and deformed RVE containing a stiff inclusion. The macroscopic kinematic data is also given. The results are coloured by the Frobenius norm of the Kirchhoff stress. The material configuration is indicated by a solid (orange) line and all results have the same geometric scale. 


\section{Discussion and conclusion}

A computational micro-to-macro transition framework for material layers possessing an energetic structure at the macroscopic scale has been presented. The continuum interface theory of Gurtin and Murdoch [1] allows the interface to undergo in-plane stretch in addition to the standard opening mode. The macroscopic kinematic descriptors of the layer's deformation state are the jump in the motion and the interface deformation gradient. The Hill-type averaging theorem was then used to identify the relations between the averaged microscopic kinetic quantities and their macroscopic counterparts. The in-plane periodicity of the heterogeneous fluctuation field was proven to be one possible restriction required to ensure satisfaction of the Hill-type averaging theorem.

The theory was then implemented using the finite element method. Following the approach of Miehe [48], developed for macroscopic material points within the bulk, it suffices to compute the reaction forces on the positive faces of the RVE in order to calculate the macroscopic kinetic quantities and tangent modulus. A series of three-dimensional numerical examples clearly illustrate the ability of the layer to stretch, and the coupling between the macroscopic kinematic and kinetic quantities.

One logical extension of this work is to implement the micro-structural problem within a fully-coupled micro-macro scheme. This intended extension would, obviously, draw heavily on the work of Hirschberger et al. [15].

Another interesting extension of this work would be to consider material surfaces with heterogeneous microstructures.

Although the model presented here significantly extends existing approaches such as [15], elastic resistance to flexure is not accounted for. The extension of the (macroscopic) Gurtin and Murdoch [1] theory to account for such resistance has been developed by Steigmann and Ogden [50] for elastic solids with energetic surfaces. Gei [51] has extended the theory of Steigmann and Ogden [50] to material interfaces. The first-order ansatz linking the macro- and microscopic scales (see Eq. (23)) would need to be extended to a second-order one in order to account for bending of the RVE. This would be done in accordance with the framework developed by Geers et al. [35-38].

\section{Acknowledgements}

The financial support of the German Science Foundation (Deutsche Forschungsgemeinschaft, DFG), grant STE 544/39-1, is gratefully acknowl- 
edged. The first author thanks the National Research Foundation of South Africa for their support. We also thank J.-P. Pelteret at the University of Cape Town for his significant contribution in the development of the finite element code.

\section{References}

[1] M. E. Gurtin, A. I. Murdoch, A continuum theory of elastic material surfaces, Archive of Rational Mechanics and Analysis 57 (1975) 291-323.

[2] R. Hill, Elastic properties of reinforced solids: Some theoretical principles, Journal of the Mechanics and Physics of Solids 11 (1963) 357-372.

[3] R. Hill, On constitutive macro-variables for heterogeneous solids at finite strain, Proceedings of the Royal Society of London. A. Mathematical and Physical Sciences 326 (1565) (1972) 131-147.

[4] P. M. Suquet, Homogenization Techniques for Composite MediaElements of homogenization of inelastic solid mechanics, in: E. Sanchez-Palencia, A. Zaoui (Eds.), Homogenization Techniques for Composite Media, Springer-Verlag, Berlin, 193-278, 1987.

[5] J.-M. Guedes, N. Kikuchi, Preprocessing and postprocessing for materials based on the homogenization method with adaptive finite element methods, Computer Methods in Applied Mechanics and Engineering 83 (2) (1990) 143-198.

[6] K. Terada, N. Kikuchi, Nonlinear homogenization method for practical applications, in: S. Ghosh, M. Ostoja-Starzewski (Eds.), Computational Methods in Micromechanics, vol. AMD-212/MD-62, ASME, 1-16, 1995.

[7] R. J. M. Smit, W. A. M. Brekelmans, H. E. H. Meijer, Prediction of the mechanical behavior of nonlinear heterogeneous systems by multi-level finite element modeling, Computer Methods in Applied Mechanics and Engineering 155 (1998) 181-192.

[8] C. Miehe, J. Schröder, J. Schotte, Computational homogenization analysis in finite plasticity. Simulation of texture development in polycrystalline materials, Computer Methods in Applied Mechanics and Engineering 171 (1999) 387-418.

[9] J. C. Michel, H. Moulinec, P. Suquet, Effective properties of composite materials with periodic microstructure: a computational approach, Computer Methods in Applied Mechanics and Engineering 172 (1-4) (1999) 109-143.

[10] F. Feyel, J.-L. Chaboche, FE2 multiscale approach for modelling the elastoviscoplastic behaviour of long fibre $\mathrm{SiC} / \mathrm{Ti}$ composite materials, Computer Methods in Applied Mechanics and Engineering 183 (3-4) (2000) 309-330.

[11] V. Kouznetsova, W. A. M. Brekelmans, F. P. T. Baaijens, An approach to micromacro modeling of heterogeneous materials, Computational Mechanics 27 (2001) 37-84.

[12] C. Miehe, Strain-driven homogenization of inelastic microstructures and composites based on an incremental variational formulation, International Journal for Numerical Methods in Engineering 55 (2002) 1285-1322.

[13] C. Miehe, A. Koch, Computational micro-to-macro transitions of discretized microstructures undergoing small strains, Archive of Applied Mechanics 72 (2002) $300-317$. 
[14] I. Temizer, P. Wriggers, On the computation of the macroscopic tangent for multiscale volumetric homogenization problems, Computer Methods in Applied Mechanics and Engineering 198 (2008) 195-210.

[15] C. B. Hirschberger, S. Ricker, P. Steinmann, N. Sukumar, Computational multiscale modelling of heterogeneous material layers, Engineering Fracture Mechanics 76 (6) (2009) 793-812.

[16] M. G. D. Geers, V. G. Kouznetsova, W. A. M. Brekelmans, Multi-scale computational homogenization: Trends and challenges, Journal of Computational and Applied Mathematics 234 (7) (2010) 2175-2182.

[17] A. Needleman, A continuum model for void nucleation by inclusion debonding, Journal of Applied Mechanics 54 (3) (1987) 525-531.

[18] X.-P. Xu, A. Needleman, Void nucleation by inclusion debonding in a crystal matrix, Modelling and Simulation in Materials Science and Engineering 1 (2) (1993) 111132.

[19] J. Mosler, I. Scheider, A thermodynamically and variationally consistent class of damage-type cohesive models, Journal of the Mechanics and Physics of Solids 59 (8) (2011) 1647-1668.

[20] K. Matouš, M. G. Kulkarni, P. H. Geubelle, Multiscale cohesive failure modeling of heterogeneous adhesives, Journal of the Mechanics and Physics of Solids 56 (4) (2008) 1511-1533.

[21] M. G. Kulkarni, P. H. Geubelle, K. Matous $\breve{s}$ Multi-scale modeling of heterogeneous adhesives: Effect of particle decohesion, Mechanics of Materials 41 (5) (2009) 573583.

[22] M. V. Cid Alfaro, A. S. J. Suiker, C. V. Verhoosel, R. de Borst, Numerical homogenization of cracking processes in thin fibre-epoxy layers, European Journal of Mechanics - A/Solids 29 (2) (2010) 119-131.

[23] M. G. Kulkarni, K. Matouš, P. H. Geubelle, Coupled multi-scale cohesive modeling of failure in heterogeneous adhesives, International Journal for Numerical Methods in Engineering 84 (8) (2010) 916-946.

[24] C. V. Verhoosel, J. J. C. Remmers, M. A. Gutiérrez, R. de Borst, Computational homogenization for adhesive and cohesive failure in quasi-brittle solids, International Journal for Numerical Methods in Engineering 83 (8-9) (2010) 1155-1179.

[25] A. I. Murdoch, A thermodynamical theory of elastic material interfaces, The Quarterly Journal of Mechanics and Applied Mathematics 29 (3) (1976) 245-275.

[26] M. E. Gurtin, A. I. Murdoch, Surface stress in solids, International Journal of Solids and Structures 14 (6) (1978) $431-440$.

[27] N. Daher, G. A. Maugin, The method of virtual power in continuum mechanics application to media presenting singular surfaces and interfaces, Acta Mechanica 60 (1986) 217-240.

[28] N. Daher, G. A. Maugin, Deformable semiconductors with interfaces: Basic continuum equations, International Journal of Engineering Science 25 (9) (1987) 10931129 .

[29] M. E. Gurtin, P. W. Voorhees, The continuum mechanics of coherent two-phase elastic solids with mass transport, Proceedings of the Royal Society: Mathematical and Physical Sciences 440 (1909) (1993) 323-343.

[30] M. E. Gurtin, Configurational Forces as Basic Concepts of Continuum Physics, in: 
Applied Mathematical Sciences, vol. 137, Springer, New York, 2000.

[31] P. Steinmann, On boundary potential energies in deformational and configurational mechanics, Journal of the Mechanics and Physics of Solids 56 (2008) 772-800.

[32] A. T. McBride, A. Javili, P. Steinmann, S. Bargmann, Geometrically nonlinear continuum thermomechanics with surface energies coupled to diffusion, Journal of the Mechanics and Physics of Solids doi:10.1016/j.jmps.2011.06.002.

[33] M. G. D. Geers, E. W. C. Coenen, V. G. Kouznetsova, Multi-scale computational homogenization of structured thin sheets, Modelling and Simulation in Materials Science and Engineering 15 (4) (2007) S393.

[34] E. W. C. Coenen, V. G. Kouznetsova, M. G. D. Geers, Computational homogenization for heterogeneous thin sheets, International Journal for Numerical Methods in Engineering 83 (8-9) (2010) 1180-1205.

[35] M. G. D. Geers, V. Kouznetsova, W. A. M. Brekelmans, Gradient-enhanced computational homogenization for the micro-macro scale transition, Journal de Physique IV 11 (PR5) (2001) Pr5-145-Pr5-152.

[36] V. Kouznetsova, M. G. D. Geers, W. A. M. Brekelmans, Multi-scale constitutive modelling of heterogeneous materials with a gradient-enhanced computational homogenization scheme, International Journal for Numerical Methods in Engineering 54 (8) (2002) 1235-1260.

[37] M. Geers, V. G. Kouznetsova, W. A. M. Brekelmans, Multiscale first-order and second-order computational homogenization of microstructures towards continua, International Journal for Multiscale Computational Engineering 1 (4) (2003) 371386.

[38] V. G. Kouznetsova, M. G. D. Geers, W. A. M. Brekelmans, Multi-scale secondorder computational homogenization of multi-phase materials: a nested finite element solution strategy, Computer Methods in Applied Mechanics and Engineering 193 (48-51) (2004) 5525-5550.

[39] P. Wriggers, J. Nettingsmeier, Homogenization and Multi-Scale Approaches for Contact Problems, in: G. Maier, F. G. Rammerstorfer, J. Salençon, P. Wriggers, T. A. Laursen (Eds.), Computational Contact Mechanics, vol. 498 of CISM Courses and Lectures, Springer Vienna, ISBN 978-3-211-77298-0, 129-161, 2007.

[40] S. Stupkiewicz, Micromechanics of Contact and Interphase Layers, Springer, Berlin Heidelberg New York, 2007.

[41] C. Truesdell, W. Noll, The Non-Linear Field Theories of Mechanics, Springer, New York, 3rd edn., 2004.

[42] M. E. Gurtin, An Introduction to Continuum Mechanics, Academic Press, Orlando, 1981.

[43] R. W. Ogden, Nonlinear Elastic Deformations, Ellis Horwood Ltd., West Susex, England, 1982.

[44] P. G. Ciarlet, Mathematical Elasticity. Volume I: Three-Dimensional Elasticity, North-Holland Publishing Company, Amsterdam, 1988.

[45] M. E. Gurtin, A. Struthers, Multiphase thermomechanics with interfacial structure 3. Evolving phase boundaries in the presence of bulk deformation, Archive for Rational Mechanics and Analysis 112 (1990) 97-160.

[46] M. E. Gurtin, J. Weissmüller, F. Larché, A general theory of curved deformable interfaces in solids at equilibrium, Philosophical Magazine A 78 (5) (1998) 1093- 
1109.

[47] G. A. Holzapfel, Nonlinear Solid Mechanics. A Continuum Approach for Engineering, John Wiley \& Sons, 2001.

[48] C. Miehe, Computational micro-to-macro transitions for discretized microstructures of heterogeneous materials at finite strains based on the minimization of averaged incremental energy, Computer Methods in Applied Mechanics and Engineering 192 (5-6) (2003) 559-591.

[49] W. Bangerth, R. Hartmann, G. Kanschat, deal.II - a general purpose object oriented finite element library, ACM Transactions on Mathematical Software 33 (4) (2007) 24.

[50] D. J. Steigmann, R. W. Ogden, Elastic surface-substrate interactions, Proceedings: Mathematical, Physical and Engineering Sciences 455 (1982) (1999) 437-474.

[51] M. Gei, Stability of a material interface, in: XVII AIMETA National Congress, Firenze, Italy, 2005. 